\title{
CONVOLUTIONS FOR ORTHOGONAL POLYNOMIALS FROM LIE AND QUANTUM ALGEBRA REPRESENTATIONS
}

\author{
H.T. KoELink And J. VAn DeR Jeugt \\ Report 96-11, Universiteit van Amsterdam
}

July 3,1996

\begin{abstract}
The interpretation of the Meixner-Pollaczek, Meixner and Laguerre polynomials as overlap coefficients in the positive discrete series representations of the Lie algebra $\mathfrak{s u}(1,1)$ and the Clebsch-Gordan decomposition leads to generalisations of the convolution identities for these polynomials. Using the Racah coefficients convolution identities for continuous Hahn, Hahn and Jacobi polynomials are obtained. From the quantised universal enveloping algebra for $\mathfrak{s u}(1,1)$ convolution identities for the Al-Salam and Chihara polynomials and the Askey-Wilson polynomials are derived by using the Clebsch-Gordan and Racah coefficients. For the quantised universal enveloping algebra for $\mathfrak{s u}(2) q$-Racah polynomials are interpreted as Clebsch-Gordan coefficients, and the linearisation coefficients for a two-parameter family of Askey-Wilson polynomials are derived.
\end{abstract}

\section{INTRODUCTION}

The representation theory of Lie algebras and quantum algebras, or quantised universal enveloping algebras [6], is intimately linked to special functions of (basic) hypergeometric type, see e.g. [24], [6]. In this paper we consider especially the Lie algebra $\mathfrak{s u}(1,1)$ and its quantum analogue $U_{q}(\mathfrak{s u}(1,1))$, and we derive convolution identities for certain orthogonal polynomials which occur as overlap coefficients. The idea, which is due to Granovskii and Zhedanov [11], see also [23], is to consider (generalised) eigenvectors of a suitable element of the Lie algebra which is a recurrence operator in an irreducible representation of this Lie algebra. Then there is a relation between these eigenvectors and the eigenvectors of this Lie algebra element in the $n$-fold tensor product of irreducible representations of the Lie algebra. From the tensor product decomposition in irreducible

1991 Mathematics Subject Classification. 33C80, 33D80, 33C45, 33D45, 17B20, $17 \mathrm{~B} 37$.

Key words and phrases. orthogonal polynomials, convolution, Lie algebra, quantum algebra.

First author is supported by the Netherlands Organization for Scientific Research (NWO) under project number 610.06.100. Second author is a Senior Research Associate of the National Fund for Scientific Research of Belgium (NFWO). 
representations for $n=2,3$, we obtain identities for these eigenvectors involving ClebschGordan and Racah coefficients. In particular, if the overlap coefficients are known in terms of special functions, we obtain identities for these special functions in this way.

For the Lie algebra $\mathfrak{s u}(1,1)$ and the positive discrete series representations a special case of this approach is contained in Granovskii and Zhedanov [11], but the result is not worked out in detail. Elaborating the method of Granovskii and Zhedanov, Van der Jeugt [23] obtains a generalisation of the classical convolution identity for the Laguerre polynomials $[9,10.12(41)]$. Van der Jeugt [23] also considers the boson Lie algebra $\mathfrak{b}(1)$, a central extension of the oscillator algebra, leading to a generalisation of the convolution identity for Hermite polynomials [9, 10.13(38)]. The last identity follows from the previous one by a well-known limit transition of Laguerre polynomials to Hermite polynomials, see e.g. [15].

Apart from the Laguerre and the Hermite polynomials, also the Meixner-Pollaczek, Meixner and Charlier polynomials, which all fit into the Askey scheme of hypergeometric orthogonal polynomials [3], [15], satisfy a convolution identity of the same form. This is a straightforward consequence of the existence of a generating function of a special kind, see Al-Salam [1]. It is also known that the Meixner-Pollaczek and the Meixner polynomials can be interpreted as overlap coefficients in the positive discrete series representations of $\mathfrak{s u}(1,1)$, see Masson and Repka [22]. In $\S 3$ we show how the method of Granovskii and Zhedanov for the two-fold tensor product of positive discrete series representations of $\mathfrak{s u}(1,1)$ leads to a generalisation of the convolution identity for Meixner-Pollaczek polynomials, from which generalised convolution formulas for Meixner, Laguerre, Charlier and Hermite polynomials can be obtained by substitution or by limit transitions. Next using the three-fold tensor product representation we obtain a very general convolution identity for continuous Hahn polynomials, and similarly for the Hahn and Jacobi polynomials. These identities can also be viewed as yielding connection coefficients between two sets of orthogonal polynomials in two variables with respect to the same orthogonality measure. With this point of view, this result coincides with Dunkl's results [7], [8]. Our derivation gives an intrinsic explanation for the occurrence of balanced ${ }_{4} F_{3}$-series as connection coefficients; they are Racah coefficients. Actually, the interpretation as orthogonal polynomials in two variables works in general, and is an intrinsic way to determine the $S$-functions in [11] and [23] in terms of orthogonal polynomials instead of reducing a triple sum to a single sum.

In $\S 4$ we apply the same idea to the quantised universal enveloping algebra $U_{q}(\mathfrak{s u}(1,1))$ and its positive discrete series representations. Due to the non-cocommutativity of the comultiplication, which is needed to define the tensor product representation, the tensor product of eigenvectors is no longer an eigenvector in the tensor product representation. This can be solved if we restrict to operators related to so-called twisted primitive elements in $U_{q}(\mathfrak{s u}(1,1))$, see e.g. [21], [17]. Then the whole machinery works and we obtain a generalisation of the Al-Salam and Chihara [2] convolution identity for the Al-Salam and Chihara polynomials by considering the Clebsch-Gordan coefficients in the two-fold tensor product. Going to the three-fold tensor product representations yields a very general convolution identity for Askey-Wilson polynomials also involving $q$-Racah polynomials, and Theorem 4.10 is the key result of this paper. Overlap coefficients are also considered 
in somewhat more generality in Klimyk and Kachurik [14], but we have to restrict ourselves to the twisted primitive elements in order to keep the action in the tensor product representations manageable.

It is interesting to note that in this derivation we have a natural interpretation of the continuous Hahn, Hahn and Jacobi polynomials as Clebsch-Gordan coefficients for the Lie algebra $\mathfrak{s u}(1,1)$. Similarly, we have an interpretation of the Askey-Wilson polynomials as Clebsch-Gordan coefficients for the quantised universal enveloping algebra $U_{q}(\mathfrak{s u}(1,1))$. In $\S 5$ we shortly discuss the corresponding result for the quantised universal enveloping algebra $U_{q}(\mathfrak{s u}(2))$, where the $q$-Racah polynomials then occur as Clebsch-Gordan coefficients. This case can be obtained formally from the results for $U_{q}(\mathfrak{s u}(1,1))$. Since in the dual Hopf $*$-algebra the so-called zonal spherical elements are known in terms of a twoparameter family of Askey-Wilson polynomials, cf. [21], we obtain the explicit linearisation coefficients for this subfamily of the Askey-Wilson polynomials.

It should be remarked that there does not seem to be an appropriate $q$-analogue of the boson Lie algebra $\mathfrak{b}(1)$. Either, the Hopf $*$-algebra structure is lacking, or, as in [13], the recurrence in the two-fold tensor product representation seems unmanageable.

Instead of using generalised eigenvectors we use the spectral theory of Jacobi matrices, which we recall briefly in $\S 2$. In particular we use this theory to interpret certain recurrence operators in $\ell^{2}\left(\mathbb{Z}_{+}\right)^{\otimes n}, n=1,2,3$, as multiplication operators in certain weighted $L^{2}$-spaces on $\mathbb{R}^{n}$. This approach exploits the theory of orthogonal polynomials, cf. Propositions 3.3 and 4.3 .

The notation for (basic) hypergeometric series is the standard one as in Gasper and Rahman [10]. Unexplained notions for quantised universal enveloping algebras can be found in Chari and Pressley [6].

Acknowledgement. We thank Tom Koornwinder for useful comments. The first author thanks the Universiteit Gent for its hospitality.

\section{JACOBI MATRICES AND ORThOGONAL POLYNOMIALS}

We recall some of the results on the spectral theory of Jacobi matrices and the relation with orthogonal polynomials. For more information we refer to Berezanskiu [4, Ch. VII, $\S 1]$, see also Masson and Repka [22], Klimyk and Kachurik [14]. The operator $J$ acting on the standard orthonormal basis $\left\{e_{n} \mid n \in \mathbb{Z}_{+}\right\}$of $\ell^{2}\left(\mathbb{Z}_{+}\right)$by

$$
J e_{n}=a_{n} e_{n+1}+b_{n} e_{n}+a_{n-1} e_{n-1}, \quad a_{n}>0, b_{n} \in \mathbb{R},
$$

is called a Jacobi matrix. This operator is symmetric, and its deficiency indices are $(0,0)$ or $(1,1)$. In particular, if the coefficients $a_{n}$ and $b_{n}$ are bounded, $J$ is a bounded operator on $\ell^{2}\left(\mathbb{Z}_{+}\right)$and thus self-adjoint. $J$ is an unbounded self-adjoint operator if $\sum_{n=0}^{\infty} a_{n}^{-1}=\infty$ by Carleman's condition. Then $e_{0}$ is a cyclic vector for $J$, i.e. the span of finite linear combinations of the form $J^{p} e_{0}, p \in \mathbb{Z}_{+}$, is dense in $\ell^{2}\left(\mathbb{Z}_{+}\right)$. This is the case for all Jacobi matrices considered in this paper.

Assuming this, we can use the same coefficients $a_{n}, b_{n}$ to generate polynomials $p_{n}(x)$ of degree $n$ in $x$ by the recurrence relation

$$
x p_{n}(x)=a_{n} p_{n+1}(x)+b_{n} p_{n}(x)+a_{n-1} p_{n-1}(x), \quad p_{-1}(x)=0, p_{0}(x)=1 .
$$


By Favard's theorem there exists a positive measure $m$ on the real line such that the polynomials $p_{n}(x)$ are orthonormal;

$$
\int_{\mathbb{R}} p_{n}(x) p_{m}(x) d m(x)=\delta_{n, m} .
$$

The measure is obtained by $m(B)=\left\langle E(B) e_{0}, e_{0}\right\rangle, B$ Borel set, where $E$ denotes the spectral decomposition of the self-adjoint operator $J$.

We can represent the operator $J$ as a multiplication operator $M_{x}$ on $L^{2}(m)$, where $M_{x} f(x)=x f(x)$. For this we define

$$
\Lambda: \ell^{2}\left(\mathbb{Z}_{+}\right) \rightarrow L^{2}(m), \quad\left(\Lambda e_{n}\right)(x)=p_{n}(x),
$$

then $\Lambda$ is a unitary operator, since it maps an orthonormal basis onto an orthonormal basis. Note that we use here that the polynomials are dense in $L^{2}(m)$, since the self-adjointness of $J$ implies that the corresponding moment problem is determined. From (2.1) and (2.2) it follows that $\Lambda \circ J=M_{x} \circ \Lambda$, so that $\Lambda$ intertwines the Jacobi matrix $J$ on $\ell^{2}\left(\mathbb{Z}_{+}\right)$with the multiplication operator $M_{x}$ on $L^{2}(m)$.

\section{THE CASE $\mathfrak{s u}(1,1)$}

The Lie algebra $\mathfrak{s u}(1,1)$ is given by

$$
[H, B]=2 B, \quad[H, C]=-2 C, \quad[B, C]=H .
$$

There is a $*$-structure by $H^{*}=H$ and $B^{*}=-C$.

The positive discrete series representations $\pi_{k}$ of $\mathfrak{s u}(1,1)$ are unitary representations labelled by $k>0$. The representation space is $\ell^{2}\left(\mathbb{Z}_{+}\right)$equipped with orthonormal basis $\left\{e_{n}^{k}\right\}_{\left\{n \in \mathbb{Z}_{+}\right\}}$. The action is given by

$$
\begin{aligned}
& \pi_{k}(H) e_{n}^{k}=2(k+n) e_{n}^{k}, \\
& \pi_{k}(B) e_{n}^{k}=\sqrt{(n+1)(2 k+n)} e_{n+1}^{k}, \\
& \pi_{k}(C) e_{n}^{k}=-\sqrt{n(2 k+n-1)} e_{n-1}^{k} .
\end{aligned}
$$

The tensor product of two positive discrete series representations decomposes as

$$
\pi_{k_{1}} \otimes \pi_{k_{2}}=\bigoplus_{j=0}^{\infty} \pi_{k_{1}+k_{2}+j}
$$

The corresponding intertwining operator can be expressed by means of the Clebsch-Gordan coefficients

$$
e_{n}^{k}=\sum_{n_{1}, n_{2}} C_{n_{1}, n_{2}, n}^{k_{1}, k_{2}, k} e_{n_{1}}^{k_{1}} \otimes e_{n_{2}}^{k_{2}}
$$

Later we also use the notation $e_{n}^{\left(k_{1} k_{2}\right) k}$ for $e_{n}^{k}$ to stress the fact that this vector arises from the decomposition $\pi_{k_{1}} \otimes \pi_{k_{2}}$ into irreducible representations. The Clebsch-Gordan coefficients are non-zero only if $n_{1}+n_{2}=n+j, k=k_{1}+k_{2}+j$ for $j, n_{1}, n_{2}, n \in \mathbb{Z}_{+}$by considering the action of $H$ on both sides. We normalise the Clebsch-Gordan coefficients by $\left\langle e_{0}^{k}, e_{0}^{k_{1}} \otimes e_{j}^{k_{2}}\right\rangle>0$.

For the above results Vilenkin and Klimyk [24, §8.7] can be consulted. 
3.1. Clebsch-Gordan coefficients and orthogonal polynomials. The Meixner-Pollaczek polynomials are defined by

$$
P_{n}^{(\lambda)}(x ; \phi)=\frac{(2 \lambda)_{n}}{n !} e_{2}^{i n \phi} F_{1}\left(\begin{array}{c}
-n, \lambda+i x \\
2 \lambda
\end{array} ; 1-e^{-2 i \phi}\right) .
$$

For $\lambda>0$ and $0<\phi<\pi$ these are orthogonal polynomials with respect to a positive measure on $\mathbb{R}$, see [15]. The orthonormal Meixner-Pollaczek polynomials

$$
p_{n}(x)=p_{n}^{(\lambda)}(x ; \phi)=\sqrt{\frac{n !}{\Gamma(n+2 \lambda)}} P_{n}^{(\lambda)}(x ; \phi)
$$

satisfy the three-term recurrence relation

$$
\begin{aligned}
2 x \sin \phi p_{n}(x) & =a_{n} p_{n+1}(x)-2(n+\lambda) \cos \phi p_{n}(x)+a_{n-1} p_{n-1}(x), \\
a_{n} & =\sqrt{(n+1)(n+2 \lambda)} .
\end{aligned}
$$

The orthogonality measure for Meixner-Pollaczek polynomials is absolutely continuous. Define

$$
w^{(\lambda)}(x ; \phi)=\frac{(2 \sin \phi)^{2 \lambda}}{2 \pi} e^{(2 \phi-\pi) x}|\Gamma(\lambda+i x)|^{2}
$$

then

$$
\int_{\mathbb{R}} p_{n}^{(\lambda)}(x ; \phi) p_{m}^{(\lambda)}(x ; \phi) w^{(\lambda)}(x ; \phi) d x=\delta_{n m}
$$

Define the self-adjoint element in $\mathfrak{s u}(1,1)$;

$$
X_{\phi}=-\cos \phi H+B-C \text {. }
$$

Proposition 3.1. $\Lambda: \ell^{2}\left(\mathbb{Z}_{+}\right) \rightarrow L^{2}\left(\mathbb{R}, w^{(k)}(x ; \phi) d x\right), e_{n}^{k} \mapsto p_{n}^{(k)}(\cdot ; \phi)$, is a unitary mapping intertwining $\pi_{k}\left(X_{\phi}\right)$ acting in $\ell^{2}\left(\mathbb{Z}_{+}\right)$with $M_{2 x \sin \phi}$ on $L^{2}\left(\mathbb{R}, w^{(k)}(x ; \phi) d x\right)$.

Here, and elsewhere, $M_{g}$ denotes multiplication by the function $g$, so $M_{g} f(x)=$ $g(x) f(x)$.

Proof. Use (3.1) and (3.5) to see that $\pi_{k}\left(X_{\phi}\right)$ is a Jacobi matrix. Next compare the coefficients with the three-term recurrence relation for the orthonormal Meixner-Pollaczek polynomials to find the result as in $\S 2$.

Proposition 3.1 states that $v^{k}(x)=\sum_{n=0}^{\infty} p_{n}^{(k)}(x ; \phi) e_{n}^{k}$ is a generalised eigenvector for $\pi_{k}\left(X_{\phi}\right)$ for the eigenvalue $2 x \sin \phi$. Next we study the action of $X_{\phi}$ in the tensor product representation $\pi_{k_{1}} \otimes \pi_{k_{2}}$. Recall that $\Delta\left(X_{\phi}\right)=1 \otimes X_{\phi}+X_{\phi} \otimes 1$. 
Proposition 3.2. $\Upsilon: \ell^{2}\left(\mathbb{Z}_{+}\right) \otimes \ell^{2}\left(\mathbb{Z}_{+}\right) \rightarrow L^{2}\left(\mathbb{R}^{2}, w^{\left(k_{1}\right)}\left(x_{1} ; \phi\right) w^{\left(k_{2}\right)}\left(x_{2} ; \phi\right) d x_{1} d x_{2}\right)$, defined by $e_{n_{1}}^{k_{1}} \otimes e_{n_{2}}^{k_{2}} \mapsto p_{n_{1}}^{\left(k_{1}\right)}\left(x_{1} ; \phi\right) p_{n_{2}}^{\left(k_{2}\right)}\left(x_{2} ; \phi\right)$ is a unitary mapping intertwining $\pi_{k_{1}} \otimes \pi_{k_{2}}\left(\Delta\left(X_{\phi}\right)\right)$ with $M_{2\left(x_{1}+x_{2}\right) \sin \phi}$.

Proof. This can be seen by using the mapping $\Lambda$ of Proposition 3.1 in the second tensor factor and solving the resulting three-term recurrence in the first factor.

Proposition 3.2 states that

$$
v^{k_{1}, k_{2}}\left(x_{1}, x_{2}\right)=\sum_{n_{1}, n_{2}=0}^{\infty} p_{n_{1}}^{\left(k_{1}\right)}\left(x_{1} ; \phi\right) p_{n_{2}}^{\left(k_{2}\right)}\left(x_{2} ; \phi\right) e_{n_{1}}^{k_{1}} \otimes e_{n_{2}}^{k_{2}}
$$

are generalised eigenvectors for $\pi_{k_{1}} \otimes \pi_{k_{2}}\left(\Delta\left(X_{\phi}\right)\right)$ for the eigenvalue $2\left(x_{1}+x_{2}\right) \sin \phi$.

So $\Upsilon$ maps the basis $e_{n_{1}}^{k_{1}} \otimes e_{n_{2}}^{k_{2}}$ onto orthonormal polynomials in two variables. By the Clebsch-Gordan decomposition (3.2) there exists another orthonormal basis $e_{n}^{k}$ for the tensor product representation space. So $\Upsilon e_{n}^{k}$ gives another set of orthonormal polynomials in two variables in $L^{2}\left(\mathbb{R}^{2}, w^{\left(k_{1}\right)}\left(x_{1} ; \phi\right) w^{\left(k_{2}\right)}\left(x_{2} ; \phi\right) d x_{1} d x_{2}\right)$.

In order to formulate the result we need the continuous Hahn polynomials [15], [16];

$$
p_{n}(x ; a, b, c, d)=i^{n} \frac{(a+c)_{n}(a+d)_{n}}{n !}{ }_{3} F_{2}\left(\begin{array}{c}
-n, n+a+b+c+d-1, a+i x \\
a+c, a+d
\end{array}\right)
$$

satisfying the orthogonality relations for $\Re(a, b, c, d)>0$

$$
\begin{array}{r}
\frac{1}{2 \pi} \int_{\mathbb{R}} \Gamma(a+i x) \Gamma(b+i x) \Gamma(c-i x) \Gamma(d-i x) p_{n}(x ; a, b, c, d) p_{m}(x ; a, b, c, d) d x= \\
\delta_{n m} \frac{\Gamma(n+a+c) \Gamma(n+a+d) \Gamma(n+b+c) \Gamma(n+b+d)}{n !(2 n+a+b+c+d-1) \Gamma(n+a+b+c+d-1)} .
\end{array}
$$

The orthogonality measure is positive for $a=\bar{c}, b=\bar{d}$.

Proposition 3.3. In $L^{2}\left(\mathbb{R}^{2}, w^{\left(k_{1}\right)}\left(x_{1} ; \phi\right) w^{\left(k_{2}\right)}\left(x_{2} ; \phi\right) d x_{1} d x_{2}\right)$ we have

$$
\begin{aligned}
\Upsilon e_{n}^{k}\left(x_{1}, x_{2}\right) & =p_{n}^{(k)}\left(x_{1}+x_{2} ; \phi\right) \Upsilon e_{0}^{k}\left(x_{1}, x_{2}\right) \\
\Upsilon e_{0}^{k}\left(x_{1}, x_{2}\right) & =C p_{j}\left(x_{1} ; k_{1}, k_{2}-i\left(x_{1}+x_{2}\right), k_{1}, k_{2}+i\left(x_{1}+x_{2}\right)\right) \\
C & =(-2 \sin \phi)^{j} \sqrt{\frac{j !\left(2 j+2 k_{1}+2 k_{2}-1\right) \Gamma\left(j+2 k_{1}+2 k_{2}-1\right)}{\Gamma\left(2 k_{1}+j\right) \Gamma\left(2 k_{2}+j\right)}}
\end{aligned}
$$

Note that $\Upsilon e_{0}^{k}\left(x_{1}, x_{2}\right)$ is indeed a polynomial in $x_{1}, x_{2}$.

Proof. The first statement follows from use of the intertwining of Proposition 3.2 and the intertwining of (3.2);

$$
2\left(x_{1}+x_{2}\right) \sin \phi \Upsilon e_{n}^{k}\left(x_{1}, x_{2}\right)=M_{2\left(x_{1}+x_{2}\right) \sin \phi} \Upsilon e_{n}^{k}\left(x_{1}, x_{2}\right)=\left(\Upsilon \pi_{k}\left(X_{\phi}\right) e_{n}^{k}\right)\left(x_{1}, x_{2}\right),
$$


which gives a three-term recurrence relation for $\Upsilon e_{n}^{k}$ with respect to $n$ of the same form as in Proposition 3.1. Taking into account the initial conditions proves the first statement.

The prove the second statement we note that for $k=k_{1}+k_{2}+j, l=k_{1}+k_{2}+i$,

$$
\begin{aligned}
\delta_{i j} \delta_{m n}=\left\langle e_{n}^{k}, e_{m}^{l}\right\rangle=\left\langle\Upsilon e_{n}^{k}\right. & \left.\Upsilon e_{m}^{l}\right\rangle=\iint_{\mathbb{R}^{2}} p_{n}^{(k)}\left(x_{1}+x_{2} ; \phi\right) p_{m}^{(l)}\left(x_{1}+x_{2} ; \phi\right) \\
& \times\left(\Upsilon e_{0}^{k}\left(x_{1}, x_{2}\right) \Upsilon e_{0}^{l}\left(x_{1}, x_{2}\right)\right) w^{\left(k_{1}\right)}\left(x_{1} ; \phi\right) w^{\left(k_{2}\right)}\left(x_{2} ; \phi\right) d x_{1} d x_{2},
\end{aligned}
$$

by the first statement and Proposition 3.2. Introduce $s=x_{1}+x_{2}, t=x_{1}$, then we find

$$
\delta_{i j} \delta_{m n}=\int_{\mathbb{R}} p_{n}^{(k)}(s ; \phi) p_{m}^{(l)}(s ; \phi) \int_{\mathbb{R}} \Upsilon e_{0}^{k}(t, s-t) \Upsilon e_{0}^{l}(t, s-t) w^{\left(k_{1}\right)}(t ; \phi) w^{\left(k_{2}\right)}(s-t ; \phi) d t d s
$$

In case $k=l$, or $i=j$, we see that the inner integral must equal the normalised orthogonality measure for the Meixner-Pollaczek polynomials $p_{n}^{(k)}(s ; \phi)$, since the corresponding moment problem is determined. In case $k \neq l$, or $i \neq j$, we conclude that the inner integral integrated against any polynomial gives zero, so that it must be zero since the polynomials are dense in $L^{2}\left(\mathbb{R}^{2}, w^{\left(k_{1}\right)}\left(x_{1} ; \phi\right) w^{\left(k_{2}\right)}\left(x_{2} ; \phi\right) d x_{1} d x_{2}\right)$. So we get

$$
\begin{aligned}
\delta_{i j} w^{(k)}(s ; \phi)=e^{(2 \phi-\pi) s} \frac{(2 \sin \phi)^{2 k_{1}+2 k_{2}}}{4 \pi^{2}} \int_{\mathbb{R}} \Upsilon e_{0}^{k}(t, s-t) \Upsilon e_{0}^{l}(t, s-t) \\
\quad \times \Gamma\left(k_{1}+i t\right) \Gamma\left(k_{2}-i s+i t\right) \Gamma\left(k_{1}-i t\right) \Gamma\left(k_{2}+i s-i t\right) d t .
\end{aligned}
$$

Apply $\Upsilon$ to (3.3) for $n=0$ to see that $\Upsilon e_{0}^{k}(t, s-t)$ is a polynomial of degree $j$ in $t$. Hence, $\Upsilon e_{0}^{k}(t, s-t)$ is a multiple of a continuous Hahn polynomial of degree $j$ with the parameters as in the proposition.

The value of the constant follows from comparing the squared norms up to a sign. The sign is determined from the condition on the Clebsch-Gordan coefficients. This implies $0<\left\langle\Upsilon e_{0}^{k}, \Upsilon e_{0}^{k_{1}} \otimes e_{j}^{k_{2}}\right\rangle$ and using the first two parts of the proposition and Proposition 3.2 shows that the sign of $C$ follows from the sign of a double integral of two orthogonal polynomials. Only the integral over $x_{2}$ is relevant, and the sign of $C$ equals the sign of the leading coefficient of the continuous Hahn polynomials viewed as a polynomial in $x_{2}$, which is $(-1)^{j}$.

So we can now apply $\Upsilon$ to (3.3) to find, $k=k_{1}+k_{2}+j$,

$$
\begin{aligned}
\sum_{n_{1}+n_{2}=n+j} C_{n_{1}, n_{2}, n}^{k_{1}, k_{2}, k} p_{n_{1}}^{\left(k_{1}\right)}\left(x_{1} ; \phi\right) p_{n_{2}}^{\left(k_{2}\right)}\left(x_{2} ; \phi\right) & =C p_{n}^{(k)}\left(x_{1}+x_{2} ; \phi\right) \\
& \times p_{j}\left(x_{1} ; k_{1}, k_{2}-i\left(x_{1}+x_{2}\right), k_{1}, k_{2}+i\left(x_{1}+x_{2}\right)\right) .
\end{aligned}
$$

The Clebsch-Gordan coefficients remain to be determined, and this can be done from this formula, see [23]. They can be expressed in terms of ${ }_{3} F_{2}$-series, which are known as Hahn polynomials. Using the Hahn polynomials defined by

$$
Q_{n}(x ; a, b, N)={ }_{3} F_{2}\left(\begin{array}{c}
-n, n+a+b+1,-x \\
a+1,-N
\end{array}\right)
$$


for $N \in \mathbb{Z}_{+}, 0 \leq n \leq N$, we have, with $k=k_{1}+k_{2}+j, n_{1}+n_{2}=n+j$,

$$
\begin{aligned}
& C_{n_{1}, n_{2}, n}^{k_{1}, k_{2}, k}=\sqrt{\frac{\left(2 k_{1}\right)_{n_{1}}\left(2 k_{2}\right)_{n_{2}}\left(2 k_{1}\right)_{j}}{n ! n_{1} ! n_{2} ! j !\left(2 k_{1}+2 k_{2}+2 j\right)_{n}\left(2 k_{2}\right)_{j}\left(2 k_{1}+2 k_{2}+j-1\right)_{j}}} \\
& \times(n+j) ! Q_{j}\left(n_{1} ; 2 k_{1}-1,2 k_{2}-1 ; n+j\right),
\end{aligned}
$$

see $[24, \S 8.7]$ for another proof.

Using this in (3.7) gives an identity in a weighted $L^{2}$-space, but since it is a polynomial identity it holds for all $x_{1}, x_{2}$. Simplifying proves the following theorem.

Theorem 3.4. With the notation for continuous Hahn, Meixner-Pollaczek and Hahn polynomials as in (3.4), (3.6) and (3.8) the following convolution formula holds:

$$
\begin{aligned}
& \left(\begin{array}{c}
n+j \\
n
\end{array}\right) \sum_{l=0}^{n+j} Q_{j}\left(l ; 2 k_{1}-1,2 k_{2}-1, n+j\right) P_{l}^{\left(k_{1}\right)}\left(x_{1} ; \phi\right) P_{n+j-l}^{\left(k_{2}\right)}\left(x_{2} ; \phi\right)= \\
& \quad \frac{(-2 \sin \phi)^{j}}{\left(2 k_{1}\right)_{j}} P_{n}^{\left(k_{1}+k_{2}+j\right)}\left(x_{1}+x_{2} ; \phi\right) p_{j}\left(x_{1} ; k_{1}, k_{2}-i\left(x_{1}+x_{2}\right), k_{1}, k_{2}+i\left(x_{1}+x_{2}\right)\right) .
\end{aligned}
$$

Remark 3.5. (i) The case $j=0$ gives back the convolution identity for the MeixnerPollaczek polynomials, see e.g. $[1, \S 8],[2]$. The case $n=0$ gives another convolution identity for Meixner-Pollaczek polynomials, since the Hahn polynomial reduces to a summable ${ }_{2} F_{1}$-series.

(ii) Note that the polynomials on both sides of the formula in Theorem 3.4 are orthogonal polynomials in two variables for the space $L^{2}\left(\mathbb{R}^{2}, w^{\left(k_{1}\right)}\left(x_{1} ; \phi\right) w^{\left(k_{2}\right)}\left(x_{2} ; \phi\right) d x_{1} d x_{2}\right)$, so we have proved a connection coefficient formula for these polynomials. The dual connection coefficient formula follows from the orthogonality of the Clebsch-Gordan matrix, or equivalently, from the orthogonality relations for the dual Hahn polynomials.

(iii) Theorem 3.4 shows that the continuous Hahn polynomials have an interpretation as Clebsch-Gordan coefficients for $\mathfrak{s u}(1,1)$. Using the generalised eigenvectors we formally have, cf. (3.3),

$$
v^{k_{1}, k_{2}}\left(x_{1}, x_{2}\right)=\sum_{k} C p_{j}\left(x_{1} ; k_{1}, k_{2}-i\left(x_{1}+x_{2}\right), k_{1}, k_{2}+i\left(x_{1}+x_{2}\right)\right) v^{k}\left(x_{1}+x_{2}\right),
$$

with $C$ as in Proposition 3.3. The dual relations can be written using the orthogonality measure for the continuous Hahn polynomials.

Recall the definition of the Laguerre polynomials $L_{n}^{(a)}(x)=(a+1)_{n} / n !{ }_{1} F_{1}(-n ; a+$ $1 ; x)$, the Jacobi polynomials $P_{n}^{(a, b)}(x)=(a+1)_{n} / n !_{2} F_{1}(-n, n+a+b+1 ; a+1 ;(1-x) / 2)$ and the Meixner polynomials $M_{n}(x ; \beta ; c)={ }_{2} F_{1}\left(-n,-x ; \beta ; 1-c^{-1}\right)$.

Corollary 3.6. (i) ([23]) The Laguerre polynomials satisfy the following convolution identity

$$
\begin{aligned}
\sum_{l=0}^{n+j} Q_{j}(l ; a, b, n+j) L_{l}^{(a)}\left(x_{1}\right) L_{n+j-l}^{(b)}\left(x_{2}\right) & =\frac{(-1)^{j} n ! j !}{(a+1)_{j}(n+j) !} \\
& \times L_{n}^{(a+b+1+2 j)}\left(x_{1}+x_{2}\right)\left(x_{1}+x_{2}\right)^{j} P_{j}^{(a, b)}\left(\frac{x_{2}-x_{1}}{x_{1}+x_{2}}\right) .
\end{aligned}
$$


(ii) The Meixner polynomials satisfy the following convolution identity

$$
\begin{aligned}
& \left(c^{-1}-1\right)^{-j} \sum_{l=0}^{n+j} \frac{(a)_{l}(b)_{n+j-l}}{l !(n+j-l) !} Q_{j}(l ; a-1, b-1, n+j) M_{l}\left(x_{1} ; a ; c\right) M_{n+j-l}\left(x_{2} ; b ; c\right)= \\
& \frac{(a+b+2 j)_{n}}{(n+j) !} M_{n}\left(x_{1}+x_{2}-j ; a+b+2 j ; c\right)\left(-x_{1}-x_{2}\right)_{j} Q_{j}\left(x_{1} ; a-1, b-1, x_{1}+x_{2}\right) .
\end{aligned}
$$

Proof. The first case follows from the limit transition of the Meixner-Pollaczek polynomials to the Laguerre polynomials; $\lim _{\phi \downarrow 0} P_{n}^{((a+1) / 2)}(-2 x / \phi ; \phi)=L_{n}^{(a)}(x)$. In this limit transition the continuous Hahn polynomials tend to the Jacobi polynomials.

The second case follows from the substitution $\phi=\ln c / 2 i$, and replacing $x_{1}$ and $x_{2}$ by $i k_{1}+i x_{1}$ and $i k_{2}+i x_{2}$. For this substitution the continuous Hahn polynomials go over into the Hahn polynomials.

Remark 3.7. (i) The case $j=0$ in both formulas gives back the convolution identities for the Laguerre and Meixner polynomials, see e.g. [1], [2], [9, 10.12(41)], and the case $n=0$ gives another convolution identity for the Laguerre and Meixner polynomials. Again these formulas can be viewed as connection coefficient formulas for orthogonal polynomials in two variables.

(ii) The identities of Corollary 3.6 can be obtained by considering the action of $X=$ $-H+B-C$ in the representations $\pi_{k}$ and $\pi_{k_{1}} \otimes \pi_{k_{2}}$ for the Laguerre case, see [23], and by considering the action of $X_{c}=-(1+c) / 2 \sqrt{c} H+B-C, 0<c<1$, in the representations $\pi_{k}$ and $\pi_{k_{1}} \otimes \pi_{k_{2}}$ for the Meixner case. The limit case $c \uparrow 1$ in the Meixner result gives the Laguerre result. In this case we can interpret the Jacobi and Hahn polynomials as Clebsch-Gordan coefficients, cf. Remark 3.5(iii).

(iii) Corollary 3.6(ii) is equivalent to Theorem 3.4 by the same substitution. Theorem 3.4 can also be obtained from Corollary 3.6(i) by a double application of the Mellin transform. For this we have to use that the Laguerre polynomials are mapped onto MeixnerPollaczek polynomials, cf. [18, §3], and that the Jacobi polynomials are mapped onto the continuous Hahn polynomials, cf. $[16,(3.4)$ with $\Gamma(\beta-i \lambda)$ replaced by $\Gamma(\beta+i \lambda)]$.

The other hypergeometric orthogonal polynomials satisfying a convolution identity are the Charlier and Hermite polynomials, cf. [1], [2]. These identities can be obtained by taking the appropriate limits from the Meixner polynomials to the Charlier polynomials and from the Laguerre polynomials to the Hermite polynomials, cf. e.g. [15]. The Hahn polynomials tend to Krawtchouk polynomials and the Jacobi polynomials tend to Hermite polynomials. We use the notation $K_{n}(x ; p, N)={ }_{2} F_{1}\left(-n,-x ;-N ; p^{-1}\right)$ for Krawtchouk polynomials, $C_{n}(x ; a)={ }_{2} F_{0}\left(-n,-x ;-; a^{-1}\right)$ for Charlier polynomials and $H_{n}(x)=(2 x)^{n}{ }_{2} F_{0}\left(-n / 2,-(n-1) / 2 ;-;-x^{-2}\right)$ for the Hermite polynomials.

Corollary 3.8. (i) ([23]) The Hermite polynomials satisfy the following convolution iden- 
tity

$$
\begin{aligned}
\sum_{l=0}^{n+j} K_{j}\left(l ; \frac{a^{2}}{a^{2}+b^{2}}, n+j\right) \frac{a^{l}}{l !} & H_{l}(x) \frac{b^{n+j-l}}{(n+j-l) !} H_{n+j-l}(y)= \\
& \frac{\left(a^{2}+b^{2}\right)^{(n+j) / 2}}{(n+j) !}\left(\frac{b}{a}\right)^{j} H_{n}\left(\frac{a x+b y}{\sqrt{a^{2}+b^{2}}}\right) H_{j}\left(\frac{a y-b x}{\sqrt{a^{2}+b^{2}}}\right) .
\end{aligned}
$$

(ii) The Charlier polynomials satisfy the following convolution identity

$$
\begin{aligned}
& \sum_{l=0}^{n+j}\left(\begin{array}{c}
n+j \\
l
\end{array}\right) \alpha^{l} \beta^{n+j-l} K_{j}\left(l ; \frac{\alpha}{\alpha+\beta}, n+j\right) C_{l}(x ; \alpha) C_{n+j-l}(y ; \beta)= \\
& (-1)^{j}(\alpha+\beta)^{n} C_{n}(x+y-j ; \alpha+\beta)(-x-y)_{j} K_{j}\left(x ; \frac{\alpha}{\alpha+\beta}, x+y\right) .
\end{aligned}
$$

Remark 3.9. (i) Again the case $j=0$ gives known convolution formulas, cf. [1, §8], [2], $[9,10.13(40)]$. Corollary 3.8 (ii) is derived in a different way in Vilenkin and Klimyk [24, $\S 8.6 .5]$.

(ii) This time the identities have a similar interpretation, but now we have to use the Lie algebra $\mathfrak{b}(1)$, a central extension of the oscillator algebra, cf. [23]. In particular we can now interpret the Hermite and Charlier polynomials as Clebsch-Gordan coefficients.

3.2. Racah coefficients and orthogonal polynomials. In the tensor product of three positive discrete series representations $\pi_{k_{1}} \otimes \pi_{k_{2}} \otimes \pi_{k_{3}}$ of $\mathfrak{s u}(1,1)$ we consider the following orthogonal bases;

$$
\begin{aligned}
e_{n}^{\left(\left(k_{1} k_{2}\right) k_{12} k_{3}\right) k} & =\sum_{n_{12}, n_{3}} C_{n_{12}, n_{3}, n}^{k_{12}, k_{3}, k} e_{n_{12}}^{\left(k_{1} k_{2}\right) k_{12}} \otimes e_{n_{3}}^{k_{3}} \\
& =\sum_{n_{1}, n_{2}, n_{3}, n_{12}} C_{n_{1}, n_{2}, n_{12}}^{k_{1}, k_{2}, k_{12}} C_{n_{12}, n_{3}, n}^{k_{12}, k_{3}, k} e_{n_{1}}^{k_{1}} \otimes e_{n_{2}}^{k_{2}} \otimes e_{n_{3}}^{k_{3}},
\end{aligned}
$$

and

$$
\begin{aligned}
e_{n}^{\left(k_{1}\left(k_{2} k_{3}\right) k_{23}\right) k} & =\sum_{n_{1}, n_{23}} C_{n_{1}, n_{23}, n}^{k_{1}, k_{23}, k} e_{n_{1}}^{k_{1}} \otimes e_{n_{23}}^{\left(k_{2} k_{3}\right) k_{23}} \\
& =\sum_{n_{1}, n_{2}, n_{3}, n_{23}} C_{n_{2}, n_{3}, n_{23}}^{k_{2}, k_{3}, k_{23}} C_{n_{1}, n_{23}, n}^{k_{1}, k_{23}, k} e_{n_{1}}^{k_{1}} \otimes e_{n_{2}}^{k_{2}} \otimes e_{n_{3}}^{k_{3}}
\end{aligned}
$$

Here we use the extended notation $e_{n}^{\left(k_{1} k_{2}\right) k}$ for the basis of the tensor product decomposition to keep track of how the decomposition is obtained.

These bases are connected by the Racah coefficients, which leads to an intertwiner for the action of $\mathfrak{s u}(1,1)$. The Racah coefficients are defined by

$$
e_{n}^{\left(\left(k_{1} k_{2}\right) k_{12} k_{3}\right) k}=\sum_{k_{23}} U_{k_{3}, k, k_{23}}^{k_{1}, k_{2}, k_{12}} e_{n}^{\left(k_{1}\left(k_{2} k_{3}\right) k_{23}\right) k} .
$$


In the previous formulas the following constraints hold

$$
\begin{gathered}
k_{12}=k_{1}+k_{2}+j_{12}, \quad k_{23}=k_{2}+k_{3}+j_{23}, \\
k=k_{12}+k_{3}+j=k_{1}+k_{23}+j^{\prime}, \quad j_{12}, j, j_{23}, j^{\prime} \in \mathbb{Z}_{+}, \text {and } j_{12}+j=j_{23}+j^{\prime} .
\end{gathered}
$$

Thus all above sums are finite sums.

Recall that $(1 \otimes \Delta)\left(\Delta\left(X_{\phi}\right)\right)=1 \otimes 1 \otimes X_{\phi}+1 \otimes X_{\phi} \otimes 1+X_{\phi} \otimes 1 \otimes 1$. The following proposition is proved as Proposition 3.2.

Proposition 3.10. Define the unitary mapping

$$
\Theta: \ell^{2}\left(\mathbb{Z}_{+}\right) \otimes \ell^{2}\left(\mathbb{Z}_{+}\right) \otimes \ell^{2}\left(\mathbb{Z}_{+}\right) \rightarrow L^{2}\left(\mathbb{R}^{3}, w^{\left(k_{1}\right)}\left(x_{1} ; \phi\right) w^{\left(k_{2}\right)}\left(x_{2} ; \phi\right) w^{\left(k_{3}\right)}\left(x_{3} ; \phi\right) d x_{1} d x_{2} d x_{3}\right)
$$

by

$$
\Theta: e_{n_{1}}^{k_{1}} \otimes e_{n_{2}}^{k_{2}} \otimes e_{n_{3}}^{k_{3}} \mapsto p_{n_{1}}^{\left(k_{1}\right)}\left(x_{1} ; \phi\right) p_{n_{2}}^{\left(k_{2}\right)}\left(x_{2} ; \phi\right) p_{n_{3}}^{\left(k_{3}\right)}\left(x_{3} ; \phi\right),
$$

then $\Theta$ intertwines $\pi_{k_{1}} \otimes \pi_{k_{2}} \otimes \pi_{k_{3}}\left((1 \otimes \Delta)\left(\Delta\left(X_{\phi}\right)\right)\right.$ with $M_{2\left(x_{1}+x_{2}+x_{3}\right) \sin \phi}$.

Remark 3.11. Let $\Lambda^{(k)}=\Lambda$ be the unitary mapping defined in Proposition 3.1 and $\Upsilon^{\left(k_{1} k_{2}\right)}=\Upsilon$ be the unitary mapping defined in Proposition 3.2. Using the identifications

$$
\begin{aligned}
L^{2}\left(\mathbb{R}^{3}, w^{\left(k_{1}\right)}\left(x_{1} ; \phi\right) w^{\left(k_{2}\right)}\left(x_{2} ; \phi\right) w^{\left(k_{3}\right)}\left(x_{3} ; \phi\right) d x_{1} d x_{2} d x_{3}\right) \\
\quad=L^{2}\left(\mathbb{R}, w^{\left(k_{1}\right)}\left(x_{1} ; \phi\right) d x_{1}\right) \otimes L^{2}\left(\mathbb{R}^{2}, w^{\left(k_{2}\right)}\left(x_{2} ; \phi\right) w^{\left(k_{3}\right)}\left(x_{3} ; \phi\right) d x_{2} d x_{3}\right) \\
\left.\quad=L^{2}\left(\mathbb{R}^{2}, w^{\left(k_{1}\right)}\left(x_{1} ; \phi\right) w^{\left(k_{2}\right)}\left(x_{2} ; \phi\right)\right) d x_{1} d x_{2}\right) \otimes L^{2}\left(\mathbb{R}, w^{\left(k_{3}\right)}\left(x_{3} ; \phi\right) d x_{3}\right),
\end{aligned}
$$

we have $\Theta=\Lambda^{\left(k_{1}\right)} \otimes \Upsilon^{\left(k_{2} k_{3}\right)}=\Upsilon^{\left(k_{1} k_{2}\right)} \otimes \Lambda^{\left(k_{3}\right)}$. Hence, for the orthogonal bases on the right hand side of (3.9) and (3.12) we have

$$
\begin{aligned}
& \Theta e_{n_{12}}^{\left(k_{1} k_{2}\right) k_{12}} \otimes e_{n_{3}}^{k_{3}}=\left(\Upsilon^{\left(k_{1} k_{2}\right)} e_{n_{12}}^{\left(k_{1} k_{2}\right) k_{12}}\right)\left(\Lambda^{\left(k_{3}\right)} e_{n_{3}}^{k_{3}}\right), \\
& \Theta e_{n_{1}}^{k_{1}} \otimes e_{n_{23}}^{\left(k_{2} k_{3}\right) k_{23}}=\left(\Lambda^{\left(k_{1}\right)} e_{n_{1}}^{k_{1}}\right)\left(\Upsilon^{\left(k_{2} k_{3}\right)} e_{n_{23}}^{\left(k_{2} k_{3}\right) k_{23}}\right) .
\end{aligned}
$$

And the right hand sides are known from Propositions 3.1 and 3.2 in terms of MeixnerPollaczek polynomials times continuous Hahn polynomials.

Proposition 3.12. (i) The following expressions hold;

$$
\begin{aligned}
& \Theta\left(e_{n}^{\left(\left(k_{1} k_{2}\right) k_{12} k_{3}\right) k}\right)\left(x_{1}, x_{2}, x_{3}\right)=p_{n}^{(k)}\left(x_{1}+x_{2}+x_{3} ; \phi\right) \Theta\left(e_{0}^{\left(\left(k_{1} k_{2}\right) k_{12} k_{3}\right) k}\right)\left(x_{1}, x_{2}, x_{3}\right), \\
& \Theta\left(e_{0}^{\left(\left(k_{1} k_{2}\right) k_{12} k_{3}\right) k}\right)\left(x_{1}, x_{2}, x_{3}\right)=\Upsilon^{\left(k_{1} k_{2}\right)} e_{0}^{\left(k_{1} k_{2}\right) k_{12}}\left(x_{1}, x_{2}\right) \Upsilon^{\left(k_{12} k_{3}\right)} e_{0}^{\left(k_{12} k_{3}\right) k}\left(x_{1}+x_{2}, x_{3}\right) .
\end{aligned}
$$

(ii) The following expressions hold;

$$
\begin{aligned}
& \Theta\left(e_{n}^{\left(k_{1}\left(k_{2} k_{3}\right) k_{23}\right) k}\right)\left(x_{1}, x_{2}, x_{3}\right)=p_{n}^{(k)}\left(x_{1}+x_{2}+x_{3} ; \phi\right) \Theta\left(e_{0}^{\left(k_{1}\left(k_{2} k_{3}\right) k_{23}\right) k}\right)\left(x_{1}, x_{2}, x_{3}\right), \\
& \Theta\left(e_{0}^{\left(k_{1}\left(k_{2} k_{3}\right) k_{23}\right) k}\right)\left(x_{1}, x_{2}, x_{3}\right)=\Upsilon^{\left(k_{2} k_{3}\right)} e_{0}^{\left(k_{2} k_{3}\right) k_{23}}\left(x_{2}, x_{3}\right) \Upsilon^{\left(k_{1} k_{23}\right)} e_{0}^{\left(k_{1} k_{23}\right) k}\left(x_{1}, x_{2}+x_{3}\right) .
\end{aligned}
$$


Proof. Statement (ii) is proved analogously as statement (i). The first statement of (i) follows from Proposition 3.10 and the decomposition of the three-fold tensor product, cf. Proposition 3.3.

For the second statement we use (3.9), Remark 3.11 and Propositions 3.3 and 3.1 to find

$$
\begin{aligned}
& \Theta\left(e_{0}^{\left(\left(k_{1} k_{2}\right) k_{12} k_{3}\right) k}\right)\left(x_{1}, x_{2}, x_{3}\right)= \\
& \left(\Upsilon^{\left(k_{1} k_{2}\right)} e_{0}^{\left(k_{1} k_{2}\right) k_{12}}\right)\left(x_{1}, x_{2}\right) \sum_{n_{12}+n_{3}=j} C_{n_{12}, n_{3}, 0}^{k_{12}, k_{3}, k} p_{n_{12}}^{\left(k_{12}\right)}\left(x_{1}+x_{2} ; \phi\right) p_{n_{3}}^{\left(k_{3}\right)}\left(x_{3} ; \phi\right) .
\end{aligned}
$$

The sum can be evaluated as $\left(\Upsilon^{\left(k_{12} k_{3}\right)} e_{0}^{\left(k_{12} k_{3}\right) k}\right)\left(x_{1}+x_{2}, x_{3}\right)$ by $(3.7)$.

Next we apply $\Theta$ to (3.13), then it follows from Proposition 3.12 that we can divide both sides by the Meixner-Pollaczek polynomial of degree $n$. Since $\Theta$ is unitary we obtain the Wigner-Eckart theorem, stating that the Racah coefficients in (3.13) are independent of $n$. So we can restrict to the case $n=0$ of (3.13) before applying $\Theta$ without loss of generality. We obtain

$$
\begin{aligned}
\sum_{j_{23}} U_{k_{3}, k, k_{23}}^{k_{1}, k_{2}, k_{12}}\left(\Upsilon^{\left(k_{2} k_{3}\right)} e_{0}^{\left(k_{2} k_{3}\right) k_{23}}\right)\left(x_{2}, x_{3}\right)\left(\Upsilon^{\left(k_{1} k_{23}\right)} e_{0}^{\left(k_{1} k_{23}\right) k}\right)\left(x_{1}, x_{2}+x_{3}\right)= \\
\left(\Upsilon^{\left(k_{1} k_{2}\right)} e_{0}^{\left(k_{1} k_{2}\right) k_{12}}\right)\left(x_{1}, x_{2}\right)\left(\Upsilon^{\left(k_{12} k_{3}\right)} e_{0}^{\left(k_{12} k_{3}\right) k}\right)\left(x_{1}+x_{2}, x_{3}\right) .
\end{aligned}
$$

The Racah coefficients remain to be determined, and this can actually be done from (3.15), see [23]. One can either copy the expression [23, (4.8)], or use the limit $q \uparrow 1$ of the expression for the $q$-Racah coefficient given in Proposition 4.9. Both lead to the following expression of the Racah coefficients in terms of balanced ${ }_{4} F_{3}$-series;

$$
\begin{gathered}
U_{k_{3}, k, k_{23}}^{k_{1}, k_{2}, k_{12}}=\left(\begin{array}{c}
j+j_{12} \\
j_{23}
\end{array}\right) \frac{\left(2 k_{2}\right)_{j_{12}}\left(2 k_{3}\right)_{j}\left(2 k_{1}+2 k_{2}+2 k_{3}+j+j_{12}-1\right)_{j_{23}}}{\left(2 k_{3}, 2 k_{2}+2 k_{3}+j_{23}-1\right)_{j_{23}}\left(2 k_{2}+2 k_{3}+2 j_{23}\right)_{j^{\prime}}} \\
\times\left(\frac{j^{\prime} !\left(2 k_{1}, 2 k_{23}, 2 k_{1}+2 k_{23}+j^{\prime}-1\right)_{j^{\prime}} j_{23} !\left(2 k_{2}, 2 k_{3}, 2 k_{2}+2 k_{3}+j_{23}-1\right)_{j_{23}}}{j !\left(2 k_{12}, 2 k_{3}, 2 k_{12}+2 k_{3}+j-1\right)_{j} j_{12} !\left(2 k_{1}, 2 k_{2}, 2 k_{1}+2 k_{2}+j_{12}-1\right)_{j_{12}}}\right)^{1 / 2} \\
\times{ }_{4} F_{3}\left(\begin{array}{c}
2 k_{1}+2 k_{2}+j_{12}-1,2 k_{2}+2 k_{3}+j_{23}-1,-j_{12},-j_{23} \\
2 k_{2}, 2 k_{1}+2 k_{2}+2 k_{3}+j+j_{12}-1,-j-j_{12}
\end{array}\right),
\end{gathered}
$$

with the convention (3.14).

The Racah coefficients can be rewritten in terms of the Racah polynomials defined by

$$
R_{n}(\lambda(x) ; \alpha, \beta, \gamma, \delta)={ }_{4} F_{3}\left(\begin{array}{c}
-n, n+\alpha+\beta+1,-x, x+\gamma+\delta+1 \\
\alpha+1, \beta+\delta+1, \gamma+1
\end{array} ; 1\right),
$$

where $\lambda(x)=x(x+\gamma+\delta+1)$, one of the lower parameters equals $-N, N \in \mathbb{Z}_{+}$and $0 \leq n \leq N$, cf. [15]. The orthogonality relations for the Racah polynomials follow from the fact that the Racah coefficients form a unitary matrix.

So we obtain the following theorem by simplifying (3.15) using $s=x_{1}+x_{2}+x_{3}$ and the explicit expression (3.16). 
Theorem 3.13. The continuous Hahn polynomials satisfy the following convolution identity

$$
\begin{aligned}
& \sum_{l=0}^{n+j}\left(\begin{array}{c}
j+n \\
n
\end{array}\right) \frac{\left(2 k_{2}\right)_{n}\left(2 k_{3}\right)_{j}\left(2 k_{1}+2 k_{2}+2 k_{3}+j+n-1\right)_{l}}{\left(2 k_{3}\right)_{l}\left(2 k_{2}+2 k_{3}+l-1\right)_{l}\left(2 k_{2}+2 k_{3}+2 l\right)_{j+n-l}} \\
& \quad \times R_{l}\left(\lambda(n) ; 2 k_{2}-1,2 k_{3}-1,-j-n-1,2 k_{1}+2 k_{2}+j+n-1\right) \\
& \quad \times p_{n+j-l}\left(x_{1} ; k_{1}, k_{2}+k_{3}+l-i s, k_{1}, k_{2}+k_{3}+l+i s\right) \\
& \quad \times p_{l}\left(x_{2} ; k_{2}, k_{3}-i\left(s-x_{1}\right), k_{2}, k_{3}+i\left(s-x_{1}\right)\right) \\
&=p_{n}\left(x_{1} ; k_{1}, k_{2}-i\left(x_{1}+x_{2}\right), k_{1}, k_{2}+i\left(x_{1}+x_{2}\right)\right) \\
& \quad \times p_{j}\left(x_{1}+x_{2} ; k_{1}+k_{2}+n, k_{3}-i s, k_{1}+k_{2}+n, k_{3}+i s\right)
\end{aligned}
$$

with the notation as in (3.6), (3.17).

Remark 3.14. (i) Theorem 3.13 can be considered as a connection coefficient formula between two systems of orthogonal polynomials for the orthogonality measure

$\Gamma\left(k_{1}+i x_{1}\right) \Gamma\left(k_{1}-i x_{1}\right) \Gamma\left(k_{2}+i x_{2}\right) \Gamma\left(k_{2}-i x_{2}\right) \Gamma\left(k_{3}+i\left(s-x_{1}-x_{2}\right)\right) \Gamma\left(k_{3}-i\left(s-x_{1}-x_{2}\right)\right) d x_{1} d x_{2}$ on $\mathbb{R}^{2}$. This follows from substituting $s=x_{1}+x_{2}+x_{3}$ in the weighted $L^{2}$-space of Proposition 3.10 and leaving out the integration with respect to $s$, which can be done by Proposition 3.12 and the Wigner-Eckart theorem.

(ii) Theorem 3.4 can be obtained as a limit case of Theorem 3.13 by letting $k_{3} \rightarrow \infty$ and using the limit transition of the continuous Hahn polynomials to the Meixner-Pollaczek polynomials, see e.g. [15]. Note that Theorem 3.4 is used in the derivation of Theorem 3.13 .

(iii) Application of $\Theta$ to (3.9) - (3.12) gives results which are immediately derivable from Theorem 3.4.

Corollary 3.15. (i) ([23]) The Jacobi polynomials satisfy the convolution identity

$$
\begin{aligned}
& \sum_{l=0}^{n+j}\left(\begin{array}{c}
j+n \\
n
\end{array}\right) \frac{(b+1)_{n}(c+1)_{j}(a+b+c+j+n+2)_{l}}{(c+1)_{l}(b+c+l+1)_{l}(b+c+2 l+2)_{j+n-l}} \\
& \quad \times R_{l}(\lambda(n) ; b, c,-j-n-1, a+b+j+n+1) \\
& \quad \times P_{n+j-l}^{(a, b+c+2 l+1)}\left(1-2 x_{1}\right)\left(1-x_{1}\right)^{l} P_{l}^{(b, c)}\left(\frac{1-x_{1}-2 x_{2}}{1-x_{1}}\right) \\
& =\left(x_{1}+x_{2}\right)^{n} P_{n}^{(a, b)}\left(\frac{x_{2}-x_{1}}{x_{1}+x_{2}}\right) P_{j}^{(a+b+2 n+1, c)}\left(1-2\left(x_{1}+x_{2}\right)\right) .
\end{aligned}
$$

(ii) The Hahn polynomials satisfy the following convolution identity

$$
\begin{aligned}
& \sum_{l=0}^{n+j}\left(\begin{array}{c}
j+n \\
l
\end{array}\right) \frac{(a+1)_{n+j-l}(b+1)_{l}(b+1)_{n}(c+1)_{j}(a+b+c+j+n+2)_{l}}{(a+1)_{n}(c+1)_{l}(b+c+l+1)_{l}(b+c+2 l+2)_{j+n-l}(a+b+2 n+2)_{j}} \\
& \quad \times R_{l}(\lambda(n) ; b, c,-j-n-1, a+b+j+n+1) \\
& \quad \times(l-s)_{n+j-l} Q_{n+j-l}\left(x_{1} ; a, b+c+2 l+1, s-l\right)\left(x_{1}-s\right)_{l} Q_{l}\left(x_{2} ; b, c, s-x_{1}\right) \\
& =\left(-x_{1}-x_{2}\right)_{n} Q_{n}\left(x_{1} ; a, b, x_{1}+x_{2}\right)(n-s)_{j} Q_{j}\left(x_{1}+x_{2}-n ; a+b+2 n+1, c, s-n\right)
\end{aligned}
$$


with the notation (3.8), (3.17).

Proof. The first result follows from the limit transition of the continuous Hahn polynomials to the Jacobi polynomials. Replace $x_{i}$ by $s x_{i}$ and let $s \rightarrow \infty$. The second result follows by a similar substitution as in the proof of Corollary 3.6(ii).

Remark 3.16. (i) Similar as in Remark 3.7(iii) we have that Corollary 3.15(ii) and Theorem 3.13 can be obtained from each other by formal substitution. Theorem 3.13 can be obtained from Corollary 3.15(i) by a double application of the Mellin transform. Moreover, Corollary 3.15 can be proved as Theorem 3.13 by analysing the action of $X$ and $X_{c}$, cf. Remark 3.7(ii), in the three-fold tensor product.

(ii) Dunkl [7, Thm. 4.2, Prop. 5.4], [8, Thm. 1.7] has obtained Corollary 3.15, and hence Theorem 3.13, by a different method. Dunkl [7] obtains the two-variable Hahn polynomials by judiciously guessing solutions for a certain difference equation arising from the representation theory of the symmetric group. By symmetry considerations there are more solutions of this type, and the connection coefficients can be calculated in terms of balanced ${ }_{4} F_{3}$-series. The derivation in this paper gives an intrinsic explanation for the occurrence of the Racah polynomials as connection coefficients. See also Dunkl [7], [8] for the orthogonality relations for these two-variable Hahn and Jacobi polynomials for suitable restrictions on the parameters.

We do not obtain extensions of Corollary 3.8 in this way. For $k_{1}, k_{2}, k_{3} \rightarrow \infty$ in Theorem 3.13 we obtain the same result. This is also explained by the fact that the Racah coefficients for the Lie algebra $\mathfrak{b}(1)$ are of the same form as the Clebsch-Gordan coefficients, cf. [23].

\section{The CASE $U_{q}(\mathfrak{s u}(1,1))$}

Let $U_{q}(\mathfrak{s l}(2, \mathbb{C}))$ be the complex unital associative algebra generated by $A, B, C, D$ subject to the relations

$$
A D=1=D A, \quad A B=q B A, \quad A C=q^{-1} C A, \quad B C-C B=\frac{A^{2}-D^{2}}{q-q^{-1}} .
$$

It is a Hopf algebra. We are only concerned with the comultiplication, which is defined by

$$
\begin{aligned}
& \Delta(A)=A \otimes A, \quad \Delta(B)=A \otimes B+B \otimes D, \\
& \Delta(C)=A \otimes C+C \otimes D, \quad \Delta(D)=D \otimes D
\end{aligned}
$$

on the level of generators and extended as an algebra homomorphism. There are several possible $*$-structures on $U_{q}(\mathfrak{s l}(2, \mathbb{C}))$, and we take

$$
A^{*}=A, \quad B^{*}=-C, \quad C^{*}=-B, \quad D^{*}=D,
$$

and the corresponding Hopf $*$-algebra is denoted by $U_{q}(\mathfrak{s u}(1,1))$. 
The positive discrete series representations $\pi_{k}$ of $U_{q}(\mathfrak{s u}(1,1))$ are unitary representations labelled by $k>0$. They act in $\ell^{2}\left(\mathbb{Z}_{+}\right)$and the action of the generators is given by

$$
\begin{aligned}
& \pi_{k}(A) e_{n}^{k}=q^{k+n} e_{n}^{k}, \\
& \pi_{k}(C) e_{n}^{k}=q^{1 / 2-k-n} \frac{\sqrt{\left(1-q^{2 n}\right)\left(1-q^{4 k+2 n-2}\right)}}{q-q^{-1}} e_{n-1}^{k}, \\
& \pi_{k}(B) e_{n}^{k}=q^{-1 / 2-k-n} \frac{\sqrt{\left(1-q^{2 n+2}\right)\left(1-q^{4 k+2 n}\right)}}{q^{-1}-q} e_{n+1}^{k} .
\end{aligned}
$$

Note that $\pi_{k}(D)$ is an unbounded operator, but that $\pi_{k}(A), \pi_{k}(B), \pi_{k}(C) \in \mathcal{B}\left(\ell^{2}\left(\mathbb{Z}_{+}\right)\right)$. The operators that we consider are bounded.

Recall that the tensor product of two representations are defined by use of the comultiplication. The tensor product of two positive discrete series representation decomposes as for the Lie algebra $\mathfrak{s u}(1,1)$;

$$
\pi_{k_{1}} \otimes \pi_{k_{2}} \cong \bigoplus_{j=0}^{\infty} \pi_{k_{1}+k_{2}+j}
$$

So there exists a unitary matrix mapping the orthogonal basis $e_{n_{1}}^{k_{1}} \otimes e_{n_{2}}^{k_{2}}$ onto $e_{n}^{k_{1}+k_{2}+j}$ intertwining the action of $U_{q}(\mathfrak{s u}(1,1))$. The matrix elements of this unitary mapping are the Clebsch-Gordan coefficients;

$$
e_{n}^{k}=\sum_{n_{1}, n_{2}=0}^{\infty} C_{n_{1}, n_{2}, n}^{k_{1}, k_{2}, k} e_{n_{1}}^{k_{1}} \otimes e_{n_{2}}^{k_{2}}
$$

where $k=k_{1}+k_{2}+j$ for $j \in \mathbb{Z}_{+}$. The sum is finite; $n_{1}+n_{2}=n+j$. The Clebsch-Gordan coefficients are normalised by $\left\langle e_{0}^{k}, e_{0}^{k_{1}} \otimes e_{j}^{k_{2}}\right\rangle>0$.

These results can be found in Burban and Klimyk [5] and Kalnins, Manocha and Miller [13]. See Chari and Pressley [6] for general information on quantised universal enveloping algebras.

4.1. Clebsch-Gordan coefficients and orthogonal polynomials. For this section we need the Askey-Wilson polynomials and the Al-Salam and Chihara polynomials, which are a subclass of the Askey-Wilson polynomials. The Askey-Wilson polynomial is defined by

$$
p_{m}(\cos \theta ; a, b, c, d \mid q)=a^{-m}(a b, a c, a d ; q)_{m 4} \varphi_{3}\left(\begin{array}{c}
q^{-m}, a b c d q^{m-1}, a e^{i \theta}, a e^{-i \theta} \\
a b, a c, a d
\end{array} ; q, q\right)
$$

and it is symmetric in its parameters $a, b, c$ and $d$, see [3]. The Al-Salam and Chihara polynomials are obtained by taking $c=d=0$ in the Askey-Wilson polynomials;

$$
s_{m}(\cos \theta ; a, b \mid q)=p_{m}(\cos \theta ; a, b, 0,0 \mid q)=a^{-m}(a b ; q)_{m 3} \varphi_{2}\left(\begin{array}{c}
q^{-m}, a e^{i \theta}, a e^{-i \theta} \\
a b, 0
\end{array} ; q, q\right)
$$


By $d m(\cdot ; a, b, c, d \mid q)$ we denote the normalised orthogonality measure for the AskeyWilson polynomials, which is absolutely continuous on $[-1,1]$ and has at most a finite number of discrete mass points outside $[-1,1]$. We put $d m(\cdot ; a, b \mid q)=d m(\cdot ; a, b, 0,0 \mid q)$ for the normalised orthogonality measure for the Al-Salam and Chihara polynomials. Explicitly, let

$$
w(z)=\frac{\left(z^{2}, z^{-2} ; q\right)_{\infty}}{(a z, a / z, b z, b / z, c z, c / z, d z, d / z ; q)_{\infty}}
$$

and we use $w(z)=w(z ; a, b, c, d \mid q)$ to stress the dependence on the parameters when needed. Let $a, b, c$ and $d$ be real, or, if complex, appearing in conjugate pairs, and let all the pairwise products of $a, b, c$ and $d$ not be greater or equal than 1 . Then the AskeyWilson polynomials $p_{n}(x)=p_{n}(x ; a, b, c, d \mid q)$ satisfy the orthogonality relations

$$
\begin{gathered}
\frac{1}{2 \pi h_{0}} \int_{0}^{\pi} p_{n}(\cos \theta) p_{m}(\cos \theta) w\left(e^{i \theta}\right) d \theta+\frac{1}{h_{0}} \sum_{k} p_{n}\left(x_{k}\right) p_{m}\left(x_{k}\right) w_{k}=\delta_{n, m} h_{n} \\
h_{n}=\frac{\left(1-q^{n-1} a b c d\right)}{\left(1-q^{2 n-1} a b c d\right)} \frac{(q, a b, a c, a d, b c, b d, c d ; q)_{n}}{(a b c d ; q)_{n}} \\
h_{0}=\frac{(a b c d ; q)_{\infty}}{(q, a b, a c, a d, b c, b d, c d ; q)_{\infty}} .
\end{gathered}
$$

The points $x_{k}$ are of the form $\frac{1}{2}\left(e q^{k}+e^{-1} q^{-k}\right)$ for $e$ any of the parameters $a, b, c$ or $d$ with absolute value greater than 1 ; the sum is over $k \in \mathbb{Z}_{+}$such that $\left|e q^{k}\right|>1$ and $w_{k}$ is the residue of $z \mapsto w(z)$ at $z=e q^{k}$ minus the residue at $z=e^{-1} q^{-k}$. So the normalised orthogonality measure $d m(\cdot ; a, b, c, d \mid q)$ can be read off from (4.8), see Askey and Wilson [3] or [10].

Let $S_{m}(x ; a, b \mid q)=s_{m}(x ; a, b \mid q) / \sqrt{(q, a b ; q)_{m}}$ denote the orthonormal Al-Salam and Chihara polynomials, which satisfy the three-term recurrence relation

$$
\begin{aligned}
2 x S_{n}(x) & =a_{n+1} S_{n+1}(x)+q^{n}(a+b) S_{n}(x)+a_{n} S_{n-1}(x), \\
a_{n} & =\sqrt{\left(1-a b q^{n-1}\right)\left(1-q^{n}\right)} .
\end{aligned}
$$

We now define

$$
Y_{s}=q^{1 / 2} B-q^{-1 / 2} C+\frac{s^{-1}+s}{q^{-1}-q}(A-D) \in U_{q}(\mathfrak{s u}(1,1)) .
$$

Then $Y_{s} A$ is a self-adjoint element in $U_{q}(\mathfrak{s u}(1,1))$ for $s \in \mathbb{R} \backslash\{0\}$, or $s \in \mathbb{T}$. $Y_{s}$ is twisted primitive element, i.e. $\Delta\left(Y_{s}\right)=A \otimes Y_{s}+Y_{s} \otimes D$, meaning that $Y_{s}$ is very much like a Lie algebra element.

We also use the notation $\mu(x)=\left(x+x^{-1}\right) / 2=\mu\left(x^{-1}\right)$ for $x \neq 0$ in this section.

Proposition 4.1. Let $\Lambda: \ell^{2}\left(\mathbb{Z}_{+}\right) \rightarrow L^{2}\left(\mathbb{R}, d m\left(\cdot ; q^{2 k} s, q^{2 k} / s \mid q^{2}\right)\right)$ be the unitary mapping defined by $\Lambda: e_{n}^{k} \mapsto S_{n}\left(\cdot ; q^{2 k} s, q^{2 k} / s \mid q^{2}\right)$, then $\Lambda$ intertwines $\pi_{k}\left(Y_{s} A\right)$ acting in $\ell^{2}\left(\mathbb{Z}_{+}\right)$with $2\left(M_{x}-\mu(s)\right) /\left(q^{-1}-q\right)$.

Proof. The bounded self-adjoint operator $\pi_{k}\left(Y_{s} A\right)$ is a Jacobi matrix by (4.10) and (4.3), and the result follows upon comparing with the three-term recurrence (4.9) for the AlSalam and Chihara polynomials as in $\S 2$. 
Proposition 4.1 says that $v^{k}(x)=\sum_{n=0}^{\infty} S_{n}\left(\mu(x) ; q^{2 k} s, q^{2 k} / s \mid q^{2}\right) e_{n}^{k}$ is a generalised eigenvector of the self-adjoint operator $\pi_{k}\left(Y_{s} A\right)$ for the eigenvalue

$$
\lambda_{x}=\frac{x+x^{-1}-s-s^{-1}}{q^{-1}-q}=2 \frac{\mu(x)-\mu(s)}{q^{-1}-q} .
$$

Due to the fact that the comultiplication on $U_{q}(\mathfrak{s u}(1,1))$ is less simple than for the Lie algebra $\mathfrak{s u}(1,1)$ it takes a little more effort to determine the action of $Y_{s} A$ in $\pi_{k_{1}} \otimes \pi_{k_{2}}$. The result can still be phrased using orthogonal polynomials in two variables.

Proposition 4.2. Define $\Upsilon: \ell^{2}\left(\mathbb{Z}_{+}\right) \otimes \ell^{2}\left(\mathbb{Z}_{+}\right) \rightarrow L^{2}\left(\mathbb{R}^{2}, d m\left(x_{1}, x_{2}\right)\right)$, where

$$
d m\left(x_{1}, x_{2}\right)=d m\left(x_{1} ; q^{2 k_{1}} w_{2}, q^{2 k_{1}} / w_{2} \mid q^{2}\right) d m\left(x_{2} ; q^{2 k_{2}} s, q^{2 k_{2}} / s \mid q^{2}\right), \quad x_{2}=\mu\left(w_{2}\right),
$$

by

$$
\Upsilon: e_{n_{1}}^{k_{1}} \otimes e_{n_{2}}^{k_{2}} \mapsto S_{n_{1}}\left(x_{1} ; q^{2 k_{1}} w_{2}, q^{2 k_{1}} / w_{2} \mid q^{2}\right) S_{n_{2}}\left(x_{2} ; q^{2 k_{2}} s, q^{2 k_{2}} / s \mid q^{2}\right)
$$

then $\Upsilon$ is a unitary mapping intertwining $\pi_{k_{1}} \otimes \pi_{k_{2}}\left(\Delta\left(Y_{s} A\right)\right)$ with $2\left(M_{x_{1}}-\mu(s)\right) /\left(q^{-1}-q\right)$ in $L^{2}\left(d m\left(x_{1}, x_{2}\right)\right)$.

Note that $\Upsilon\left(e_{n_{1}}^{k_{1}} \otimes e_{n_{2}}^{k_{2}}\right)$ forms a set of orthogonal polynomials in two variables $x_{1}$ and $x_{2}$ for $L^{2}\left(\mathbb{R}^{2}, d m\left(x_{1}, x_{2}\right)\right)$, since the Al-Salam and Chihara polynomial is symmetric in its parameters.

Proposition 4.2 states that the vector

$$
\begin{aligned}
w\left(x_{1} ; x_{2}\right) & =\sum_{n_{1}=0}^{\infty} S_{n_{1}}\left(\mu\left(x_{1}\right) ; q^{2 k_{1}} x_{2}, q^{2 k_{1}} / x_{2} \mid q^{2}\right) e_{n_{1}}^{k_{1}} \otimes v^{k_{2}}\left(x_{2}\right) \\
& =\sum_{n_{1}, n_{2}=0}^{\infty} S_{n_{1}}\left(\mu\left(x_{1}\right) ; q^{2 k_{1}} x_{2}, q^{2 k_{1}} / x_{2} \mid q^{2}\right) S_{n_{2}}\left(\mu\left(x_{2}\right) ; q^{2 k_{2}} s, q^{2 k_{2}} / s \mid q^{2}\right) e_{n_{1}}^{k_{1}} \otimes e_{n_{2}}^{k_{2}}
\end{aligned}
$$

is a generalised eigenvector of $\pi_{k_{1}} \otimes \pi_{k_{2}}\left(\Delta\left(Y_{s} A\right)\right)$ for the eigenvalue $\lambda_{x_{1}}$. This last observation is essentially the way to obtain Proposition 4.2 , since $\Delta\left(Y_{s} A\right)=A^{2} \otimes Y_{s} A+Y_{s} A \otimes 1$ acts as a three-term recurrence operator in $e_{n_{1}}^{k_{1}} \otimes v^{k_{2}}\left(x_{2}\right)$.

Proof. We use $\Delta\left(Y_{s} A\right)=A^{2} \otimes Y_{s} A+Y_{s} A \otimes 1$ and Proposition 4.1 to define for fixed $x_{2}$ the $\operatorname{map} \Lambda_{0}: \ell^{2}\left(\mathbb{Z}_{+}\right) \otimes \ell^{2}\left(\mathbb{Z}_{+}\right) \rightarrow \ell^{2}\left(\mathbb{Z}_{+}\right)$by

$$
\Lambda_{0}: e_{n_{1}}^{k_{1}} \otimes e_{n_{2}}^{k_{2}} \mapsto S_{n_{2}}\left(x_{2} ; q^{2 k_{2}} s, q^{2 k_{2}} / s \mid q^{2}\right) e_{n_{1}}^{k_{1}}
$$

to obtain the recurrence in $n_{1}$

$$
\begin{aligned}
& \Lambda_{0}\left(\left(q^{-1}-q\right)\left(\pi_{k_{1}} \otimes \pi_{k_{2}}\left(\Delta\left(Y_{s} A\right)\right)+s+s^{-1}\right) e_{n_{1}}^{k_{1}} \otimes e_{n_{2}}^{k_{2}}=\right. \\
& \quad S_{n_{2}}\left(x_{2} ; q^{2 k_{2}} s, q^{2 k_{2}} / s \mid q^{2}\right)\left(q^{2 n_{1}}\left(\left(s+s^{-1}\right) q^{2 k_{1}}+\lambda_{w_{2}} q^{2 k_{1}}\left(q^{-1}-q\right)\right) e_{n_{1}}^{k_{1}}\right. \\
& \left.\quad+\sqrt{\left(1-q^{2 n_{1}+2}\right)\left(1-q^{4 k_{1}+2 n}\right)} e_{n_{1}+1}^{k_{1}}+\sqrt{\left(1-q^{2 n_{1}}\right)\left(1-q^{4 k_{1}+2 n_{1}-1}\right)} e_{n_{1}-1}^{k_{1}}\right)
\end{aligned}
$$

Use the explicit expression for $\lambda_{w_{2}}$ and the three-term recurrence relation (4.9) to obtain the result.

We now calculate the action of $\Upsilon e_{n}^{k}$, which yields another set of orthonormal polynomials for $L^{2}\left(\mathbb{R}^{2}, d m\left(x_{1}, x_{2}\right)\right)$. 
Proposition 4.3. Let $k=k_{1}+k_{2}+j$ for $j \in \mathbb{Z}_{+}$, and $x_{1}=\mu\left(w_{1}\right)$ then

$$
\begin{aligned}
\left(\Upsilon e_{n}^{k}\right)\left(x_{1}, x_{2}\right) & =S_{n}\left(x_{1} ; q^{2 k} s, q^{2 k} / s \mid q^{2}\right)\left(\Upsilon e_{0}^{k}\right)\left(x_{1}, x_{2}\right), \\
\left(\Upsilon e_{0}^{k}\right)\left(x_{1}, x_{2}\right) & =C p_{j}\left(x_{2} ; q^{2 k_{1}} w_{1}, q^{2 k_{1}} / w_{1}, q^{2 k_{2}} s, q^{2 k_{2}} / s \mid q^{2}\right), \\
C^{-1}=\left(\left(C_{j}\left(k_{1}, k_{2}\right)\right)^{-1}\right. & =\sqrt{\left(q^{2}, q^{4 k_{1}}, q^{4 k_{2}}, q^{4 k_{1}+4 k_{2}+2 j-2} ; q^{2}\right)_{j}} .
\end{aligned}
$$

Proof. By Proposition 4.2, (4.4) and

$$
2 \frac{x_{1}-\mu(s)}{q^{-1}-q} \Upsilon e_{n}^{k}\left(x_{1}, x_{2}\right)=\Upsilon\left(\pi_{k}\left(Y_{s} A\right) e_{n}^{k}\right)\left(x_{1}, x_{2}\right)
$$

we obtain the three-term recurrence relation as in Proposition 4.1, but with different initial conditions. Hence, the first statement follows.

Since $\Upsilon$ is unitary we have the orthogonality relations $\delta_{n m} \delta_{k l}=\left\langle\Upsilon e_{n}^{k}, \Upsilon e_{m}^{l}\right\rangle=$

$$
\int S_{n}\left(x_{1} ; q^{2 k} s, q^{2 k} / s \mid q^{2}\right) S_{m}\left(x_{1} ; q^{2 l} s, q^{2 l} / s \mid q^{2}\right) \int \Upsilon e_{0}^{k}\left(x_{1}, x_{2}\right) \Upsilon e_{0}^{l}\left(x_{1}, x_{2}\right) d m\left(x_{1}, x_{2}\right),
$$

by our first observation. As in the proof of Proposition 3.3 we conclude $\Upsilon e_{0}^{k}\left(x_{1}, x_{2}\right)=$ $p_{j}\left(x_{2}\right)$ is polynomial of degree $j, k=k_{1}+k_{2}+j$, in $x_{2}$ satisfying the orthogonality relations

$$
\int_{x_{2}} p_{j}\left(x_{2}\right) p_{i}\left(x_{2}\right) d m\left(x_{1}, x_{2}\right)=\delta_{i j} d m\left(x_{1} ; q^{2 k} s, q^{2 k} / s \mid q^{2}\right)
$$

as measures with respect to functions in the variable $x_{1}$.

We now assume for ease of presentation that $d m\left(x_{1}, x_{2}\right)$ is absolutely continuous. The general case can be proved similarly, or it can be obtained by analytic continuation with respect to $s$. The measure is absolutely continuous for $q^{2 k_{2}}<|s|<q^{-2 k_{2}}$, since $k_{1}, k_{2}>0$. Put $x_{1}=\cos \theta, x_{2}=\cos \psi$, then we obtain the explicit expression (4.8) for the orthogonality measure;

$$
\begin{aligned}
& \frac{1}{2 \pi} \int_{0}^{\pi} p_{i}(\cos \psi) p_{j}(\cos \psi) \frac{\left(e^{ \pm 2 i \psi}, e^{ \pm 2 i \theta} ; q^{2}\right)_{\infty}}{\left(q^{2 k_{2}} s e^{ \pm i \psi}, q^{2 k_{2}} e^{ \pm i \psi} / s, q^{2 k_{1}} e^{ \pm i \psi \pm i \theta} ; q^{2}\right)_{\infty}} d \psi= \\
& \delta_{i j} \frac{\left(q^{4 k_{1}+4 k_{2}+4 j} ; q^{2}\right)_{\infty}}{\left(q^{2}, q^{4 k_{1}}, q^{4 k_{2}} ; q^{2}\right)_{\infty}} \frac{\left(e^{ \pm 2 i \theta} ; q^{2}\right)_{\infty}}{\left(q^{2 k_{1}+2 k_{2}+2 j} s e^{ \pm i \theta}, q^{2 k_{1}+2 k_{2}+2 j} e^{ \pm i \theta} / s ; q^{2}\right)_{\infty}}
\end{aligned}
$$

for almost all $\theta$. The \pm -signs means that we take all possible combinations in the infinite $q$ shifted factorials. Cancelling the $\left(e^{ \pm 2 i \theta} ; q^{2}\right)_{\infty}$ on both sides and comparing the result with (4.8) we see that $p_{j}$ is a multiple of $p_{j}\left(\cdot ; q^{2 k_{1}} e^{i \theta}, q^{2 k_{1}} e^{-i \theta}, q^{2 k_{2}} s, q^{2 k_{2}} / s \mid q^{2}\right)$. The constant in front follows up to a sign by comparing the squared norms. As in the proof of Proposition 3.3 the sign of $C$ follows from the normalisation of the Clebsch-Gordan coefficients, and now we obtain $C>0$.

So we obtain a second set of orthonormal polynomials for $L^{2}\left(\mathbb{R}^{2}, d m\left(x_{1}, x_{2}\right)\right)$ in terms of Al-Salam and Chihara polynomials and Askey-Wilson polynomials. 
The convolution formula for the Al-Salam and Chihara polynomials is obtained by applying $\Upsilon$ to (4.5) using the results of Propositions 4.2 and 4.3. The results holds as an identity in a weighted $L^{2}$-space, but since it is a polynomial identity it holds for all $x_{1}, x_{2}$; with $x_{1}=\mu\left(w_{1}\right), x_{2}=\mu\left(w_{2}\right)$, and $k=k_{1}+k_{2}+j$

$$
\begin{gathered}
\sum_{n_{1}+n_{2}=n+j} C_{n_{1}, n_{2}, n}^{k_{1}, k_{2}, k} S_{n_{1}}\left(x_{1} ; q^{2 k_{1}} w_{2}, q^{2 k_{1}} / w_{2} \mid q^{2}\right) S_{n_{2}}\left(x_{2} ; q^{2 k_{2}} s, q^{2 k_{2}} / s \mid q^{2}\right)= \\
\frac{S_{n}\left(x_{1} ; q^{2 k} s, q^{2 k} / s \mid q^{2}\right) p_{j}\left(x_{2} ; q^{2 k_{1}} w_{1}, q^{2 k_{1}} / w_{1}, q^{2 k_{2}} s, q^{2 k_{2}} / s \mid q^{2}\right)}{\sqrt{\left(q^{2}, q^{4 k_{1}}, q^{4 k_{2}}, q^{4 k_{1}+4 k_{2}+2 j-2} ; q^{2}\right)_{j}}}
\end{gathered}
$$

We have not yet calculated the Clebsch-Gordan coefficients explicitly, but we can now use (4.11) to determine $C_{n_{1}, n_{2}, n}^{k_{1}, k_{2}, k}$ by specialising to a generating function for the ClebschGordan coefficients. The result is phrased in terms of $q$-Hahn polynomials, which are defined as follows;

$$
Q_{n}\left(q^{-x} ; a, b, N ; q\right)={ }_{3} \varphi_{2}\left(\begin{array}{c}
q^{-n}, q^{-x}, a b q^{n+1} \\
a q, q^{-N}
\end{array} ; q, q\right)
$$

See e.g. [13] for other derivations of the following lemma.

Lemma 4.4. With $n_{1}+n_{2}=n+j$ we get

$$
C_{n_{1}, n_{2}, n}^{k_{1}, k_{2}, k_{1}+k_{2}+j}=C Q_{j}\left(q^{-2 n_{1}} ; q^{4 k_{1}-2}, q^{4 k_{2}-2}, n+j ; q^{2}\right)
$$

with the constant $C$ given by

$$
\frac{q^{2 k_{1}\left(n-n_{1}\right)}\left(q^{2} ; q^{2}\right)_{n+j} \sqrt{\left(q^{4 k_{1}} ; q^{2}\right)_{n_{1}}\left(q^{4 k_{2}} ; q^{2}\right)_{n_{2}}\left(q^{4 k_{1}} ; q^{2}\right)_{j}}}{\sqrt{\left(q^{2} ; q^{2}\right)_{n}\left(q^{2} ; q^{2}\right)_{n_{1}}\left(q^{2} ; q^{2}\right)_{n_{2}}\left(q^{2} ; q^{2}\right)_{j}\left(q^{4 k_{1}+4 k_{2}+4 j} ; q^{2}\right)_{n}\left(q^{4 k_{2}} ; q^{2}\right)_{j}\left(q^{4 k_{1}+4 k_{2}+2 j-2} ; q^{2}\right)_{j}}} .
$$

Proof. Observe that $C_{n_{1}, n_{2}, n}^{k_{1}, k_{2}, k}$ is independent of $s, x_{1}=\mu\left(w_{1}\right)$ and $x_{2}=\mu\left(w_{2}\right)$. Specialise $w_{2}=q^{2 k_{2}} s$ and $w_{1}=q^{2 k_{1}} / w_{2}=q^{2 k_{1}-2 k_{2}} / s$, then the Al-Salam and Chihara polynomials in the summand on the left hand side of (4.11) can be evaluated explicitly, since the ${ }_{3} \varphi_{2}$ series reduces to 1. For this choice the Askey-Wilson polynomial on the right hand side can also be evaluated explicitly, and we obtain the generating function for the Clebsch-Gordan coefficients

$$
\begin{aligned}
& \sum_{n_{1}+n_{2}=n+j} C_{n_{1}, n_{2}, n}^{k_{1}, k_{2}, k} q^{2 n_{1}\left(k_{2}-k_{1}\right)-2 n_{2} k_{2}} s^{n_{1}-n_{2}} \frac{\sqrt{\left(q^{4 k_{1}} ; q^{2}\right)_{n_{1}}\left(q^{4 k_{2}} ; q^{2}\right)_{n_{2}}}}{\sqrt{\left(q^{2} ; q^{2}\right)_{n_{1}}\left(q^{2} ; q^{2}\right)_{n_{2}}}}= \\
& \frac{q^{-2 j k_{2}-2 n\left(k_{1}+k_{2}+j\right)} s^{n-j}\left(q^{4 k_{1}}, q^{4 k_{2}}, q^{4 k_{2}} s^{2} ; q^{2}\right)_{j}}{\sqrt{\left(q^{2}, q^{4 k_{1}}, q^{4 k_{2}}, q^{4 k_{1}+4 k_{2}+2 j-2} ; q^{2}\right)_{j}}} \sqrt{\frac{\left(q^{4 k_{1}+4 k_{2}+4 j} ; q^{2}\right)_{n}}{\left(q^{2} ; q^{2}\right)_{n}}}
\end{aligned}
$$

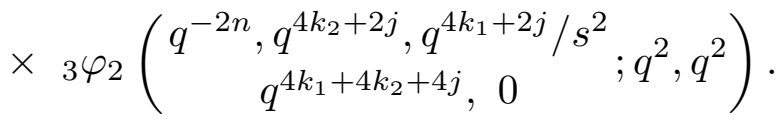


This determines $C_{n_{1}, n_{2}, n}^{k_{1}, k_{2}, k}$, but it takes some work to find the expression in terms of $q$ Hahn polynomials. First, take $n_{1}$ as the summation parameter in the sum and multiply both sides by $s^{n+j}$ to find that both sides are polynomials of degree $n+j$ in $s^{2}$. Apply [10, (III.6)] to rewrite the ${ }_{3} \varphi_{2}$-series as a polynomial in $s^{2}$ and the $q$-binomial theorem [10, (II.3)] to write $\left(q^{4 k_{2}} s^{2} ; q^{2}\right)_{j}$ as a polynomial in $s^{2}$. Comparing next the coefficients on both sides gives an expression for the Clebsch-Gordan coefficients as a terminating ${ }_{3} \varphi_{2}$-series. To put it into the required form in terms of $q$-Hahn polynomials, we need to apply some transformations for ${ }_{3} \varphi_{2}$-series, namely [10, (III.13), (III.11)]. The constant follows by a straightforward calculation.

Combining Lemma 4.4 with the unitarity of the intertwining operator consisting of the Clebsch-Gordan coefficients results in the orthogonality relations for the $q$-Hahn and dual $q$-Hahn polynomials, cf. [10, $\S 7.2]$.

We now have all ingredients to rewrite (4.11). Simplifying proves the following theorem.

Theorem 4.5. With the notation (4.7) and (4.6) for the Al-Salam and Chihara polynomials and Askey-Wilson polynomials and $x_{1}=\mu\left(w_{1}\right), x_{2}=\mu\left(w_{2}\right), n, j \in \mathbb{Z}_{+}, k_{1}, k_{2}>0$ we have

$$
\begin{gathered}
\left(q^{4 k_{1}} ; q^{2}\right)_{j} \sum_{l=0}^{n+j} q^{2 k_{1}(n-l)}\left[\begin{array}{c}
n+j \\
l
\end{array}\right]_{q^{2}} Q_{j}\left(q^{-2 l} ; q^{4 k_{1}-2}, q^{4 k_{2}-2}, n+j ; q^{2}\right) \\
\quad \times s_{l}\left(x_{1} ; q^{2 k_{1}} w_{2}, q^{2 k_{1}} / w_{2} \mid q^{2}\right) s_{n+j-l}\left(x_{2} ; q^{2 k_{2}} s, q^{2 k_{2}} / s \mid q^{2}\right)= \\
s_{n}\left(x_{1} ; q^{2 k_{1}+2 k_{2}+2 j} s, q^{2 k_{1}+2 k_{2}+2 j} / s \mid q^{2}\right) p_{j}\left(x_{2} ; q^{2 k_{1}} w_{1}, q^{2 k_{1}} / w_{1}, q^{2 k_{2}} s, q^{2 k_{2}} / s \mid q^{2}\right) .
\end{gathered}
$$

Remark 4.6. (i) Theorem 4.5 is a connection coefficient formula for orthogonal polynomials in two variables, orthogonal for the same measure, where the connection coefficients are given by the $q$-Hahn polynomials. The dual connection coefficient problem follows from the orthogonality for the Clebsch-Gordan coefficients or, equivalently, from the orthogonality relations for the dual $q$-Hahn polynomials.

(ii) The case $j=0$ gives a simple convolution property for the Al-Salam and Chihara polynomials, since the $q$-Hahn and the Askey-Wilson polynomial reduce to 1 . This was the motivation for Al-Salam and Chihara [2] to introduce the Al-Salam and Chihara polynomials as the most general set of orthogonal polynomials still satisfying a convolution property, see also Al-Salam $[1, \S 8]$. The case $n=0$ is also of interest, since then the $q$-Hahn polynomial can be evaluated and the Al-Salam and Chihara polynomial on the right hand side reduces to 1 . In both cases we have a free parameter in the sum.

(iii) Formally, in the representation space $\ell^{2}\left(\mathbb{Z}_{+}\right) \otimes \ell^{2}\left(\mathbb{Z}_{+}\right)$we have two bases of (generalised) eigenvectors for the action of $Y_{s} A$, namely $v^{k}(x)$ and $w\left(x_{1} ; x_{2}\right)$. They are connected by Clebsch-Gordan coefficients, which are now expressible as Askey-Wilson polynomials;

$$
w\left(x_{1} ; x_{2}\right)=\sum_{j=0}^{\infty} \frac{p_{j}\left(\mu\left(x_{2}\right) ; q^{2 k_{1}} x_{1}, q^{2 k_{1}} / x_{1}, q^{2 k_{2}} s, q^{2 k_{2}} / s \mid q^{2}\right)}{\sqrt{\left(q^{2}, q^{4 k_{1}}, q^{4 k_{2}}, q^{4 k_{1}+4 k_{2}+2 j-2} ; q^{2}\right)_{j}}} v^{k_{1}+k_{2}+j}\left(x_{1}\right) .
$$

The dual Clebsch-Gordan coefficient relation follows by integrating against the appropriate orthogonality measure for the Askey-Wilson polynomials. 
4.2. Racah coefficients and orthogonal polynomials. In the tensor product of three positive discrete series representations $\pi_{k_{1}} \otimes \pi_{k_{2}} \otimes \pi_{k_{3}}$ of $U_{q}(\mathfrak{s u}(1,1))$ we have the same orthogonal bases as in $\S 3.2$ and we use the same notation as in (3.9)- (3.12). Similarly, we now have an intertwiner for the $U_{q}(\mathfrak{s u}(1,1))$-action in terms of $q$-Racah coefficients;

$$
e_{n}^{\left(\left(k_{1} k_{2}\right) k_{12} k_{3}\right) k}=\sum_{k_{23}} U_{k_{3}, k, k_{23}}^{k_{1}, k_{2}, k_{12}} e_{n}^{\left(k_{1}\left(k_{2} k_{3}\right) k_{23}\right) k} .
$$

Again the constraints (3.14) hold.

Proposition 4.7. Define $\Theta: \ell^{2}\left(\mathbb{Z}_{+}\right) \otimes \ell^{2}\left(\mathbb{Z}_{+}\right) \otimes \ell^{2}\left(\mathbb{Z}_{+}\right) \rightarrow L^{2}\left(\mathbb{R}^{3}, d m\left(x_{1}, x_{2}, x_{3}\right)\right)$ by

$$
\begin{aligned}
& \Theta\left(e_{n_{1}}^{k_{1}} \otimes e_{n_{2}}^{k_{2}} \otimes e_{n_{3}}^{k_{3}}\right)\left(x_{1}, x_{2}, x_{3}\right) \\
& \quad=S_{n_{1}}\left(x_{1} ; q^{2 k_{1}} w_{2}, q^{2 k_{1}} / w_{2} \mid q^{2}\right) S_{n_{2}}\left(x_{2} ; q^{2 k_{2}} w_{3}, q^{2 k_{2}} / w_{3} \mid q^{2}\right) S_{n_{3}}\left(x_{3} ; q^{2 k_{3}} s, q^{2 k_{3}} / s \mid q^{2}\right)
\end{aligned}
$$

with the measure $\operatorname{dm}\left(x_{1}, x_{2}, x_{3}\right)$ given by

$$
d m\left(x_{1} ; q^{2 k_{1}} w_{2}, q^{2 k_{1}} / w_{2} \mid q^{2}\right) d m\left(x_{2} ; q^{2 k_{2}} w_{3}, q^{2 k_{2}} / w_{3} \mid q^{2}\right) d m\left(x_{3} ; q^{2 k_{3}} s, q^{2 k_{3}} / s \mid q^{2}\right)
$$

where $x_{i}=\mu\left(w_{i}\right)$. Then $\Theta$ is a unitary map intertwining $\pi_{k_{1}} \otimes \pi_{k_{2}} \otimes \pi_{k_{3}}\left((1 \otimes \Delta)\left(\Delta\left(Y_{s} A\right)\right)\right.$ with $2\left(M_{x_{1}}-\mu(s)\right) /\left(q^{-1}-q\right)$.

Proof. Observe that $(1 \otimes \Delta)\left(\Delta\left(Y_{s} A\right)\right)=A^{2} \otimes \Delta\left(Y_{s} A\right)+Y_{s} A \otimes \Delta(1)$. The proof now proceeds as the proof of Proposition 4.2.

Proposition 4.8. (i) The following equality holds with $x_{i}=\mu\left(w_{i}\right)$;

$$
\begin{gathered}
\Theta\left(e_{n}^{\left(\left(k_{1} k_{2}\right) k_{12} k_{3}\right) k}\right)\left(x_{1}, x_{2}, x_{3}\right)=C_{j}\left(k_{12}, k_{3}\right) C_{j_{12}}\left(k_{1}, k_{2}\right) S_{n}\left(x_{1} ; q^{2 k} s, q^{2 k} / s \mid q^{2}\right) \\
\times p_{j}\left(x_{3} ; q^{2 k_{12}} w_{1}, q^{2 k_{12}} / w_{1}, q^{2 k_{3}} s, q^{2 k_{3}} / s \mid q^{2}\right) p_{j_{12}}\left(x_{2} ; q^{2 k_{1}} w_{1}, q^{2 k_{1}} / w_{1}, q^{2 k_{2}} w_{3}, q^{2 k_{2}} / w_{3} \mid q^{2}\right) .
\end{gathered}
$$

(ii) The following equality holds with $x_{i}=\mu\left(w_{i}\right)$;

$$
\begin{gathered}
\Theta\left(e_{n}^{\left(k_{1}\left(k_{2} k_{3}\right) k_{23}\right) k}\right)\left(x_{1}, x_{2}, x_{3}\right)=C_{j^{\prime}}\left(k_{1}, k_{23}\right) C_{j_{23}}\left(k_{2}, k_{3}\right) S_{n}\left(x_{1} ; q^{2 k} s, q^{2 k} / s \mid q^{2}\right) \\
\times p_{j^{\prime}}\left(x_{2} ; q^{2 k_{1}} w_{1}, q^{2 k_{1}} / w_{1}, q^{2 k_{23}} s, q^{2 k_{23}} / s \mid q^{2}\right) p_{j_{23}}\left(x_{3} ; q^{2 k_{2}} w_{2}, q^{2 k_{2}} / w_{2}, q^{2 k_{3}} s, q^{2 k_{23}} / s \mid q^{2}\right) .
\end{gathered}
$$

The constant $C_{j}\left(k_{1}, k_{2}\right)$ is defined in Proposition 4.3.

The proof of Proposition 4.8 is slightly more complicated than the proof of its counterpart Proposition 3.12 due to the fact that we do not have a nice factorisation for $\Theta$ as in Remark 3.11. This is a consequence of the non-cocommutativity of the comultiplication for $U_{q}(\mathfrak{s u}(1,1))$.

Note that the occurrence of $S_{n}\left(x_{1} ; q^{2 k} s, q^{2 k} / s \mid q^{2}\right)$ on the right hand side corresponds to the intertwining property of the Racah coefficients as in the proof of the first statement of Proposition 4.3. 
Proof. The proof of (i) and (ii) is similar. To prove (i) we use $(3.10)$ (for the $U_{q}(\mathfrak{s u}(1,1))$ setting) and Proposition 4.7 to find

$$
\begin{aligned}
& \Theta\left(e_{n}^{\left(\left(k_{1} k_{2}\right) k_{12} k_{3}\right) k}\right)\left(x_{1}, x_{2}, x_{3}\right)=\sum_{n_{12}+n_{3}=n+j} C_{n_{12}, n_{3}, n}^{k_{12}, k_{3}, k} S_{n_{3}}\left(x_{3} ; q^{2 k_{3}} s, q^{2 k_{3}} / s \mid q^{2}\right) \\
& \times \sum_{n_{1}+n_{2}=n_{12}+j_{12}} C_{n_{1}, n_{2}, n_{12}}^{k_{1}, k_{2}, k_{12}} S_{n_{1}}\left(x_{1} ; q^{2 k_{1}} w_{2}, q^{2 k_{1}} / w_{2} \mid q^{2}\right) S_{n_{2}}\left(x_{2} ; q^{2 k_{2}} w_{3}, q^{2 k_{2}} / w_{3} \mid q^{2}\right) \\
& =C_{j_{12}}\left(k_{1}, k_{2}\right) p_{j_{12}}\left(x_{2} ; q^{2 k_{1}} w_{1}, q^{2 k_{1}} / w_{1}, q^{2 k_{2}} w_{3}, q^{2 k_{2}} / w_{3} \mid q^{2}\right) \\
& \quad \times \sum_{n_{12}+n_{3}=n+j} C_{n_{12}, n_{3}, n}^{k_{12}, k_{3}, k} S_{n_{3}}\left(x_{3} ; q^{2 k_{3}} s, q^{2 k_{3}} / s \mid q^{2}\right) S_{n_{12}}\left(x_{1} ; q^{2 k_{12}} w_{3}, q^{2 k_{12}} / w_{3}, \mid q^{2}\right)
\end{aligned}
$$

by (4.11). The last sum can be evaluated by another application of (4.11) leading to the result.

The $n$-dependence in the right hand sides of Proposition 4.8 is the same, so we obtain the Wigner-Eckhart theorem for the $U_{q}(\mathfrak{s u}(1,1))$-setting by applying $\Theta$ to (4.12). So we can restrict to $n=0$ in (4.12) before applying $\Theta$ without loss of generality, and we obtain the following polynomial identity in $x_{2}$ and $x_{3}$ with $w_{1}$ as a parameter;

$$
C_{j}\left(k_{12}, k_{3}\right) C_{j_{12}}\left(k_{1}, k_{2}\right) p_{j}\left(x_{3} ; q^{2 k_{12}} w_{1}, q^{2 k_{12}} / w_{1}, q^{2 k_{3}} s, q^{2 k_{3}} / s \mid q^{2}\right)
$$

$$
\begin{aligned}
& \times p_{j_{12}}\left(x_{2} ; q^{2 k_{1}} w_{1}, q^{2 k_{1}} / w_{1}, q^{2 k_{2}} w_{3}, q^{2 k_{2}} / w_{3} \mid q^{2}\right)=\sum_{j_{23}=0}^{j_{12}+j} U_{k_{3}, k, k_{23}}^{k_{1}, k_{2}, k_{12}} C_{j^{\prime}}\left(k_{1}, k_{23}\right) C_{j_{23}}\left(k_{2}, k_{3}\right) \\
& \times p_{j^{\prime}}\left(x_{2} ; q^{2 k_{1}} w_{1}, q^{2 k_{1}} / w_{1}, q^{2 k_{23}} s, q^{2 k_{23}} / s \mid q^{2}\right) p_{j_{23}}\left(x_{3} ; q^{2 k_{2}} w_{2}, q^{2 k_{2}} / w_{2}, q^{2 k_{3}} s, q^{2 k_{23}} / s \mid q^{2}\right) .
\end{aligned}
$$

Again we can use (4.13) in two ways. Firstly, we specialise to a suitable formula from which the Racah coefficients can be determined explicitly. Secondly, with the explicit expression for the Racah coefficients we derive a convolution identity for the Askey-Wilson polynomials.

Proposition 4.9. The Racah coefficients of (4.12) are given by

$$
U_{k_{3}, k, k_{23}}^{k_{1}, k_{2}, k_{12}}=C_{4} \varphi_{3}\left(\begin{array}{r}
q^{4 k_{1}+4 k_{2}+2 j_{12}-2}, q^{4 k_{2}+4 k_{3}+2 j_{23}-2}, q^{-2 j_{12}}, q^{-2 j_{23}} \\
q^{4 k_{2}}, q^{4 k_{1}+4 k_{2}+4 k_{3}+2 j+2 j_{12}-2}, q^{-2 j-2 j_{12}}
\end{array} ; q^{2} ; q^{2}\right),
$$

with the constant $C$ given by

$$
\begin{gathered}
\frac{\left(q^{2}, q^{4 k_{1}}, q^{4 k_{23}}, q^{4 k_{1}+4 k_{23}+2\left(j+j_{12}-j_{23}\right)-2} ; q^{2}\right)_{j+j_{12}-j_{23}}^{1 / 2}\left(q^{2}, q^{4 k_{2}}, q^{4 k_{3}}, q^{4 k_{2}+4 k_{3}+2 j_{23}-2} ; q^{2}\right)_{j_{23}}^{1 / 2}}{\left(q^{2}, q^{4 k_{12}}, q^{4 k_{3}}, q^{4 k_{12}+4 k_{3}+2 j-2} ; q^{2}\right)_{j}^{1 / 2}\left(q^{2}, q^{4 k_{1}}, q^{4 k_{2}}, q^{4 k_{1}+4 k_{2}+2 j_{12}-2} ; q^{2}\right)_{j_{12}}^{1 / 2}} \\
\times q^{2 k_{2}\left(j-j_{23}\right)}\left[\begin{array}{c}
j+j_{12} \\
j_{23}
\end{array}\right]_{q^{2}} \frac{\left(q^{4 k_{3}} ; q^{2}\right)_{j}\left(q^{4 k_{2}} ; q^{2}\right)_{j_{12}}\left(q^{4 k_{1}+4 k_{2}+4 k_{3}+2 j+2 j_{12}-2} ; q^{2}\right)_{j_{23}}}{\left(q^{4 k_{3}}, q^{4 k_{2}+4 k_{3}+2 j_{23}-2} ; q^{2}\right)_{j_{23}}\left(q^{4 k_{2}+4 k_{3}+4 j_{23}} ; q^{2}\right)_{j+j_{12}-j_{23}}} .
\end{gathered}
$$

Proof. Take $w_{1}=s=1$ and $x_{2}=\mu\left(q^{2 k_{1}}\right)$ in (4.13) to find

$$
\begin{aligned}
& p_{j}\left(x_{3} ; q^{2 k_{12}}, q^{2 k_{12}}, q^{2 k_{3}}, q^{2 k_{3}} \mid q^{2}\right)\left(q^{2 k_{1}+2 k_{2}} w_{3}, q^{2 k_{1}+2 k_{2}} / w_{3} ; q^{2}\right)_{j_{12}} \\
&=\sum_{j_{23}} C_{1} U_{k_{3}, k, k_{23}}^{k_{1}, k_{2}, k_{12}} p_{j_{23}}\left(x_{3} ; q^{2 k_{1}+2 k_{2}}, q^{2 k_{2}-2 k_{1}}, q^{2 k_{3}}, q^{2 k_{3}} \mid q^{2}\right)
\end{aligned}
$$


for $C_{1}$ an explicit constant depending upon $k_{1}, k_{2}, k_{3}, j_{12}, j_{23}$ and $j$, since two AskeyWilson polynomials can be evaluated for this choice. So the Racah coefficients occur as the coefficients when developing the polynomial of degree $j+j_{12}$ on the left hand side into Askey-Wilson polynomials. Hence the Racah coefficients can be obtained from

$$
\begin{aligned}
& C_{2} U_{k_{3}, k, k_{23}}^{k_{1}, k_{2}, k_{12}}= \\
& \qquad p_{j_{23}}\left(x_{3} ; q^{2 k_{1}+2 k_{2}}, q^{2 k_{2}-2 k_{1}}, q^{2 k_{3}}, q^{2 k_{3}} \mid q^{2}\right) p_{j}\left(x_{3} ; q^{2 k_{12}}, q^{2 k_{12}}, q^{2 k_{3}}, q^{2 k_{3}} \mid q^{2}\right) \\
& \quad \times\left(q^{2 k_{1}+2 k_{2}} w_{3}, q^{2 k_{1}+2 k_{2}} / w_{3} ; q^{2}\right)_{j_{12}} d m\left(x_{3} ; q^{2 k_{1}+2 k_{2}}, q^{2 k_{2}-2 k_{1}}, q^{2 k_{3}}, q^{2 k_{3}} \mid q^{2}\right),
\end{aligned}
$$

for some known constant $C_{2}$. Now observe that

$$
\begin{aligned}
\left(q^{2 k_{1}+2 k_{2}} w_{3}, q^{2 k_{1}+2 k_{2}} / w_{3} ; q^{2}\right)_{j_{12}} d m\left(x_{3} ; q^{2 k_{1}+2 k_{2}}, q^{2 k_{2}-2 k_{1}}, q^{2 k_{3}}, q^{2 k_{3}} \mid q^{2}\right) \\
=C_{3} d m\left(x_{3} ; q^{2 k_{12}}, q^{2 k_{2}-2 k_{1}}, q^{2 k_{3}}, q^{2 k_{3}} \mid q^{2}\right)
\end{aligned}
$$

for some constant known $C_{3}$ by (3.14) and (4.8). Thus the Racah coefficients can be obtained by integration;

$$
\begin{aligned}
& C_{4} U_{k_{3}, k, k_{23}}^{k_{1}, k_{2}, k_{12}}=\int p_{j_{23}}\left(x_{3} ; q^{2 k_{1}+2 k_{2}}, q^{2 k_{2}-2 k_{1}}, q^{2 k_{3}}, q^{2 k_{3}} \mid q^{2}\right) \\
& \quad \times p_{j}\left(x_{3} ; q^{2 k_{12}}, q^{2 k_{12}}, q^{2 k_{3}}, q^{2 k_{3}} \mid q^{2}\right) d m\left(w_{3} ; q^{2 k_{12}}, q^{2 k_{2}-2 k_{1}}, q^{2 k_{3}}, q^{2 k_{3}} \mid q^{2}\right),
\end{aligned}
$$

with $C_{4}$ explicitly known. Observe that three out of four of the parameters of each of the Askey-Wilson polynomials in (4.14) coincide with the parameters of the Askey-Wilson measure in (4.14). Use the connection coefficient formula for Askey-Wilson polynomials with one different parameter, cf. Askey and Wilson $[3,(6.4-5)]$ or see $[10,(7.6 .8-9)$ with the right hand side of $(7.6 .9)$ multiplied by $\left.(q ; q)_{n}\right]$, twice to rewrite the Askey-Wilson polynomials in terms of Askey-Wilson polynomials with the same parameters as the AskeyWilson measure in (4.14). By orthogonality the integration is then easily performed and we are left with a single sum, which can be written as a very-well-poised ${ }_{8} \varphi_{7}$-series. This can be transformed to a balanced ${ }_{4} \varphi_{3}$-series by Watson's transformation [10, (III.17)], and another application of Sears's transformation [10, (III.15)] gives the form as in the statement of the proposition. The constant follows from bookkeeping.

Recall the $q$-Racah polynomials, see $[10, \S 7.2]$, [15],

$$
R_{n}(\nu(x) ; \alpha, \beta, \gamma, \delta ; q)={ }_{4} \varphi_{3}\left(\begin{array}{c}
q^{-n}, \alpha \beta q^{n+1}, q^{-x}, \gamma \delta q^{x+1} \\
\alpha q, \beta \delta q, \gamma q
\end{array} ; q, q\right)
$$

with $\nu(x)=q^{-x}+\gamma \delta q^{x+1}$, one of the lower parameters equals $q^{-N}, N \in \mathbb{Z}_{+}$, and $0 \leq n \leq$ $N$. The ${ }_{4} \varphi_{3}$-series in Proposition 4.9 can be written in terms of a $q$-Racah polynomial.

We can now rewrite (4.13) to arrive at the key result of this paper. For convenience we replace $q^{2}$ by $q,(a, b, c)=\left(q^{k_{1}}, q^{k_{2}}, q^{k_{3}}\right)$, and we relabel $w_{1}, j_{23}$ and $j_{12}$ by $t, l$ and $n$, and finally replace $x_{2}, x_{3}$ by $x_{1}, x_{2}$. We obtain the following $q$-analogue of Theorem 3.13 and Corollary 3.15. 
Theorem 4.10. With $x_{1}=\mu\left(w_{1}\right), x_{2}=\mu\left(w_{2}\right), n, j \in \mathbb{Z}_{+}$, we have the convolution identity for the Askey-Wilson polynomials

$$
\begin{aligned}
& \sum_{l=0}^{n+j} b^{j-l}\left[\begin{array}{c}
j+n \\
l
\end{array}\right]_{q} \frac{\left(b^{2} ; q\right)_{n}\left(a^{2} b^{2} c^{2} q^{j+n-1} ; q\right)_{l}\left(c^{2} ; q\right)_{j}}{\left(c^{2}, b^{2} c^{2} q^{l-1} ; q\right)_{l}\left(b^{2} c^{2} q^{2 l} ; q\right)_{j+n-l}} \\
& \quad \times R_{l}\left(\nu(n) ; b^{2} / q, c^{2} / q, q^{-j-n-1}, a^{2} b^{2} q^{n+j-1} ; q\right) \\
& \quad \times p_{j+n-l}\left(x_{1} ; a t, a / t, b c q^{l} s, b c q^{l} / s \mid q\right) p_{l}\left(x_{2} ; b w_{1}, b / w_{1}, c s, c / s \mid q\right) \\
& =p_{n}\left(x_{1} ; a t, a / t, b w_{2}, b / w_{2} \mid q\right) p_{j}\left(x_{2} ; a b q^{n} t, a b q^{n} / t, c s, c / s \mid q\right),
\end{aligned}
$$

with the notation of (4.6), (4.15).

Remark 4.11. (i) Theorem 4.5 can be obtained as a special case of Theorem 4.10 by letting $c \rightarrow 0$.

(ii) Theorem 4.10 leads to a kind of generating function for the $q$-Racah and $q$-Hahn polynomials. Choosing $w_{1}=a t$ and $w_{2}=c s$ in Theorem 4.10 reduces all four AskeyWilson polynomials to a single term. The remaining free parameters $s$ and $t$ appear only in the combination $s / t$. Replacing $b c s /(a t)$ by $u$, and $\left(a^{2}, b^{2}, c^{2}\right)$ by $(\alpha, \beta, \gamma)$ gives:

$$
\begin{aligned}
& \sum_{l=0}^{n+j}\left[\begin{array}{c}
j+n \\
l
\end{array}\right]_{q} \frac{(\alpha ; q)_{j+n-l}(\beta ; q)_{n}\left(\alpha \beta \gamma q^{j+n-1} ; q\right)_{l}}{(\alpha ; q)_{n}\left(\beta \gamma q^{l-1} ; q\right)_{l}\left(\beta \gamma q^{2 l} ; q\right)_{j+n-l}} u^{j-l}\left(\beta \gamma q^{l} / u ; q\right)_{j+n-l}(u ; q)_{l} \\
& \quad \times R_{l}\left(\nu(n) ; \beta / q, \gamma / q, q^{-j-n-1}, \alpha \beta q^{n+j-1} ; q\right)=\left(\alpha q^{n} u ; q\right)_{j}(\beta / u ; q)_{n} .
\end{aligned}
$$

For $\gamma=0$ we obtain a similar identity for $q$-Hahn polynomials:

$$
\sum_{l=0}^{n+j}\left[\begin{array}{c}
j+n \\
l
\end{array}\right]_{q} \frac{(\alpha ; q)_{j+n-l}(\beta ; q)_{n}}{(\alpha ; q)_{n}} Q_{n}\left(q^{-l} ; \beta / q, \alpha / q, n+j ; q\right) u^{j-l}(u ; q)_{l}=\left(\alpha q^{n} u ; q\right)_{j}(\beta / u ; q)_{n} .
$$

(iii) Theorem 4.10 gives the connection coefficients for two sets of orthogonal polynomials with respect to the absolutely continuous measure

$$
\frac{\left(w_{1}^{ \pm 2}, w_{2}^{ \pm 2} ; q\right)_{\infty}}{\left(t a w_{1}^{ \pm 1}, a w_{1}^{ \pm 1} / t, c s w_{2}^{ \pm 1}, c w_{2}^{ \pm 1} / s ; q\right)_{\infty}} \frac{1}{\left(b w_{1}^{ \pm 1} w_{2}^{ \pm 1} ; q\right)_{\infty}} \frac{d w_{1}}{w_{1}} \frac{d w_{2}}{w_{2}}
$$

on the torus $\mathbb{T}^{2}$ for $|t|^{-1}<|a|<|t|,|s|^{-1}<|c|<|s|$ and $|b|<1$. Here all possible signs for \pm have to be used. (Otherwise discrete masses at points and lines have to be added.) This weight function is invariant under simultaneously interchanging $w_{1}$ with $w_{2}, t$ with $s$ and $a$ and $c$. This transforms the orthogonal polynomials on the right hand side of Theorem 4.10 to the ones occurring in the left hand side.

In particular, note that for $s=t, a=c$, the weight function is invariant under the Weyl group for $B_{2}$, i.e. the group generated by $\left(w_{1}, w_{2}\right) \mapsto\left(w_{2}, w_{1}\right)$ and $\left(w_{1}, w_{2}\right) \mapsto\left(w_{1}, w_{2}^{-1}\right)$. The corresponding Weyl group invariant orthogonal polynomials are

$$
\begin{aligned}
p_{n}\left(\mu\left(w_{1}\right) ; a t, a / t, b w_{2}, b / w_{2} \mid q\right) p_{j}\left(\mu\left(w_{2}\right) ; a b q^{n} t, a b q^{n} / t, a t, a / t \mid q\right) \\
\quad+p_{n}\left(\mu\left(w_{2}\right) ; a t, a / t, b w_{1}, b / w_{1} \mid q\right) p_{j}\left(\mu\left(w_{1}\right) ; a b q^{n} t, a b q^{n} / t, a t, a / t \mid q\right)
\end{aligned}
$$

for $n \geq j \geq 0$. These orthogonal polynomials do not seem directly related to the Koornwinder-Macdonald orthogonal polynomials associated with root system $B C_{2}$, see [20], although the structure of the orthogonality measure is similar. 


\section{LineARISATION COEFFICIENTS FOR ASKEY-WiLsON POLYNOMIALS}

In the results of the previous section using $U_{q}(\mathfrak{s u}(1,1))$, especially Theorems 4.5 and 4.10, we can use analytic continuation with respect to the parameters involved to find similar identities but with the Al-Salam and Chihara polynomials and the Askey-Wilson polynomials replaced by the dual $q$-Krawtchouk polynomials and the $q$-Racah polynomials. These identities can be obtained by the same procedure using $U_{q}(\mathfrak{s u}(2))$ and its representation theory instead of using $U_{q}(\mathfrak{s u}(1,1))$. In particular we can now give an interpretation for $q$-Racah polynomials as Clebsch-Gordan coefficients for $U_{q}(\mathfrak{s u}(2))$. In this case we also have some knowledge on the structure of the dual Hopf $*$-algebra and this can be used to obtain a linearisation formula for a two-parameter family of Askey-Wilson polynomials. This is an application of the results of the previous section.

We first recall $U_{q}(\mathfrak{s u}(2))$ and its representation theory, see e.g. [6], [17], [21]. The Hopf algebra structure on $U_{q}(\mathfrak{s u}(2))$ is the same as the Hopf algebra structure on $U_{q}(\mathfrak{s l}(2, \mathbb{C}))$, cf. (4.1), (4.2). The *-operator making $U_{q}(\mathfrak{s u}(2))$ into a Hopf $*$-algebra is given by

$$
A^{*}=A, \quad B^{*}=C, \quad C^{*}=B, \quad D^{*}=D .
$$

There is precisely one irreducible unitary $U_{q}(\mathfrak{s u}(2))$-module $W_{N}$ of each dimension $N+1$ with highest weight vector $v_{+}$, i.e. $A v_{+}=q^{N / 2} v_{+}, B v_{+}=0$. The corresponding representation is denoted by $t^{N}$. With respect to the standard orthonormal basis $e_{n}^{N}$, $0 \leq n \leq N$, the action of the generators is given by $t^{N}(A) e_{n}^{N}=q^{n-N / 2} e_{d}^{N}$ and

$$
\begin{aligned}
& t^{N}(B) e_{n}^{N}=\frac{q^{(1-N) / 2}}{1-q^{2}} \sqrt{\left(1-q^{2 n+2}\right)\left(1-q^{2 N-2 n}\right)} e_{n+1}^{N} \\
& t^{N}(C) e_{n}^{N}=\frac{q^{(1-N) / 2}}{1-q^{2}} \sqrt{\left(1-q^{2 n}\right)\left(1-q^{2 N-2 n+2}\right)} e_{n-1}^{N}
\end{aligned}
$$

with the convention $e_{-1}^{N}=0=e_{N+1}^{N}$. So $e_{N}^{N}$ is the highest weight vector. The representation $t^{N}$, considered as a representation of $U_{q}(\mathfrak{s l}(2, \mathbb{C}))$ can be obtained from the discrete series representation $\pi_{k}$ of (4.3) by formally replacing $k$ by $-N / 2$.

The Clebsch-Gordan decomposition holds; as unitary $U_{q}(\mathfrak{s u}(2))$-modules

$$
W_{N_{1}} \otimes W_{N_{2}}=\bigoplus_{j=0}^{\min \left(N_{1}, N_{2}\right)} W_{N_{1}+N_{2}-2 j}
$$

The matrix coefficients of the intertwining operator give the Clebsch-Gordan coefficients;

$$
e_{n}^{N}=\sum_{n_{1}, n_{2}} C_{n_{1}, n_{2}, n}^{N_{1}, N_{2}, N} e_{n_{1}}^{N_{1}} \otimes e_{n_{2}}^{N_{2}}
$$

Of course, the Clebsch-Gordan coefficient is zero if $N \neq N_{1}+N_{2}-2 j$ for $0 \leq j \leq$ $\min \left(N_{1}, N_{2}\right)$. By considering the action of $A$ on both sides we see that the Clebsch-Gordan coefficient is zero unless $n_{1}+n_{2}=n+j$, so that the sum is actually a single sum. The 
Clebsch-Gordan coefficients are normalised by $\left\langle e_{0}^{N}, e_{j}^{N_{1}} \otimes e_{0}^{N_{2}}\right\rangle>0$ if $N=N_{1}+N_{2}-2 j$, $0 \leq j \leq \min \left(N_{1}, N_{2}\right)$.

We are particularly interested in the element

$$
X_{p}=q^{1 / 2} B+q^{-1 / 2} C-\frac{p^{1 / 2}-p^{-1 / 2}}{q-q^{-1}}(A-D) \in U_{q}(\mathfrak{s l}(2, \mathbb{C})), \quad p>0 .
$$

Then $X_{p} A$ is self-adjoint and $\Delta\left(X_{p} A\right)=A^{2} \otimes X_{p} A+X_{p} A \otimes 1$. Koornwinder [21] has shown that in each module $W_{N}$ the action of $X_{p} A$ is completely diagonalisable. To formulate this result we introduce the orthonormal dual $q$-Krawtchouk polynomials; for $a>0$,

$$
r_{n}\left(q^{-x}-q^{x-N} / a ; a, N ; q\right)=(-1)^{n} a^{n / 2} q^{n(n-1) / 4}\left[\begin{array}{c}
N \\
n
\end{array}\right]_{q}^{1 / 2} R_{n}\left(q^{-x}-q^{x-N} / a ; a, N ; q\right)
$$

for $N \in \mathbb{Z}_{+}$and $0 \leq x, n \leq N$. The dual $q$-Krawtchouk polynomials are defined by

$$
R_{n}\left(q^{-x}-q^{x-N} / a ; a, N ; q\right)={ }_{3} \varphi_{2}\left(\begin{array}{c}
q^{-n}, q^{-x},-q^{x-N} / a \\
q^{-N}, 0
\end{array} ; q, q\right) .
$$

The corresponding three-term recurrence relation is

$$
\begin{aligned}
\left(q^{-x}-q^{x-N} / a\right) r_{n} & =A_{n} r_{n+1}+q^{n-N}\left(1-a^{-1}\right) r_{n}+A_{n-1} r_{n-1} \\
A_{n} & =a^{-1 / 2} q^{-N+n / 2} \sqrt{\left(1-q^{n+1}\right)\left(1-q^{N-n}\right)}
\end{aligned}
$$

for $0 \leq x, n \leq N$ and $r_{n}=r_{n}\left(q^{-x}-q^{x-N} / a ; a, N ; q\right)$.

Proposition 5.1. ([21]) There exists an orthogonal basis $\phi_{f}^{N}=\phi_{f}^{N}(p), 0 \leq f \leq N$, of $W_{N}$ of eigenvectors of $t^{N}\left(X_{p} A\right)$ for the eigenvalue

$$
\lambda_{f}^{N}(p)=\frac{p^{1 / 2} q^{N-2 f}-p^{-1 / 2} q^{2 f-N}+p^{-1 / 2}-p^{1 / 2}}{q^{-1}-q} .
$$

Moreover, $\phi_{f}^{N}(p)=\sum_{n=0}^{N} r_{n}\left(q^{-2 f}-q^{2 f-2 N} / p ; p, N ; q^{2}\right) e_{d}^{N}$.

Proposition 5.1 is the analogue of Proposition 4.1, and we could have formulated it in a similar fashion using the finite discrete orthogonality measure for the dual $q$-Krawtchouk polynomials. Actually, replacing $e^{i \theta}, k$ in $S_{n}\left(\cos \theta ; q^{2 k} s, q^{2 k} / s \mid q^{2}\right)$ by $q^{-2 f+N} / s,-N / 2$ and next taking $s^{2}=-p^{-1}$ gives $r_{n}\left(q^{-2 f}-q^{2 f-2 N} / p ; p, N ; q^{2}\right)$. The analogue of Proposition 4.2 is the following.

Proposition 5.2. For $0 \leq f_{1} \leq N_{1}, 0 \leq f_{2} \leq N_{2}$ define in $W_{N_{1}} \otimes W_{N_{2}}$ the vector

$$
\phi_{f_{1}, f_{2}}^{N_{1}, N_{2}}=\sum_{n_{1}=0}^{N_{1}} r_{n_{1}}\left(q^{-2 f_{1}}-q^{2 f_{1}-2 N_{1}-2 N_{2}+4 f_{2}} / p ; p q^{2 N_{2}-4 f_{2}}, N_{1} ; q^{2}\right) e_{n_{1}}^{N_{1}} \otimes \phi_{f_{2}}^{N_{2}},
$$


then $t^{N_{1}} \otimes t^{N_{2}}\left(\Delta\left(X_{p} A\right)\right) \phi_{f_{1}, f_{2}}^{N_{1}, N_{2}}=\lambda_{f_{1}+f_{2}}^{N_{1}+N_{2}}(p) \phi_{f_{1}, f_{2}}^{N_{1}, N_{2}}$. Moreover, $\phi_{f_{1}, f_{2}}^{N_{1}, N_{2}}, 0 \leq f_{1} \leq N_{1}$, $0 \leq f_{2} \leq N_{2}$, constitutes an orthogonal basis of $W_{N_{1}} \otimes W_{N_{2}}$ of eigenvectors of $\Delta\left(X_{p} A\right)$.

Proof. From $\Delta\left(X_{p} A\right)=A^{2} \otimes X_{p} A+X_{p} A \otimes 1$ it follows that there is an eigenvector of the form $\sum_{n_{1}=0}^{N_{1}} p_{n_{1}} e_{n_{1}}^{N_{1}} \otimes \phi_{f_{2}}^{N_{2}}$ by solving a three-term recurrence relation for the $p_{n_{1}}$. Now (5.2) can be used to solve this.

There are $\left(N_{1}+1\right)\left(N_{2}+1\right)$ eigenvectors in $W_{N_{1}} \otimes W_{N_{2}}$ and $\left\langle\phi_{f_{1}, f_{2}}^{N_{1}, N_{2}}, \phi_{g_{1}, g_{2}}^{N_{1}, N_{2}}\right\rangle$ equals zero if $f_{2} \neq g_{2}$ by Proposition 5.1 and it also equals zero if $f_{1}+f_{2} \neq g_{1}+g_{2}$ by the self-adjointness of $X_{p} A$.

The result of Proposition 5.2 can be obtained from Proposition 4.2 by substituting $k_{1}, k_{2}$ by $-N_{1} / 2,-N_{2} / 2$ and $w_{1}, w_{2}$ by $q^{N_{1}+N_{2}-2 f_{1}-2 f_{2}} / s, q^{N_{2}-2 f_{2}} / s$ and $s^{2}$ by $-p^{-1}$.

Proposition 5.3. For $N=N_{1}+N_{2}-2 j, 0 \leq j \leq \min \left(N_{1}, N_{2}\right)$ we have

$$
\left\langle\phi_{f_{1}, f_{2}}^{N_{1}, N_{2}}, e_{n}^{N}\right\rangle=r_{n}\left(q^{2 j-2 f_{1}-2 f_{2}}-q^{2 f_{1}+2 f_{2}-2 j-2 N} / p ; p, N ; q^{2}\right)\left\langle\phi_{f_{1}, f_{2}}^{N_{1}, N_{2}}, e_{0}^{N}\right\rangle,
$$

if $0 \leq f_{1}+f_{2}-j \leq N$, and $\left\langle\phi_{f_{1}, f_{2}}^{N_{1}, N_{2}}, e_{n}^{N}\right\rangle=0$ otherwise. If non-zero, then

$$
\begin{aligned}
\left\langle\phi_{f_{1}, f_{2}}^{N_{1}, N_{2}}, e_{0}^{N}\right\rangle & =\left[\begin{array}{c}
N_{2} \\
j
\end{array}\right]_{q^{2}}^{1 / 2} \frac{p^{j / 2} q^{j\left(2 N_{1}+N_{2}\right)} q^{-3 j(j-1) / 2}}{\sqrt{\left(q^{2 N_{1}}, q^{2 N_{1}+2 N_{2}-2 j+2} ; q^{-2}\right)_{j}}}\left(-p^{-1} q^{2 f_{1}+2 f_{2}-2 N_{1}-2 N_{2}} ; q^{2}\right)_{j} \\
& \times\left(q^{-2 f_{1}-2 f_{2}} ; q^{2}\right)_{j} \varphi_{3}\left(\begin{array}{c}
q^{-2 j}, q^{2 j-2-2 N_{1}-2 N_{2}}, q^{-2 f_{2}},-p^{-1} q^{2 f_{2}-2 N_{2}} \\
q^{-2 N_{2}}, q^{-2 f_{1}-2 f_{2}},-p^{-1} q^{2 f_{1}+2 f_{2}-2 N_{1}-2 N_{2}} ; q^{2}, q^{2}
\end{array}\right) .
\end{aligned}
$$

Note that the ${ }_{4} \varphi_{3}$-series is balanced, and can be written in terms of $q$-Racah polynomials (4.15). The ${ }_{4} \varphi_{3}$-series in Proposition 5.3 equals

$$
R_{j}\left(q^{-2 f_{2}}-p^{-1} q^{2 f_{2}-2 N_{2}} ; q^{-2 N_{2}-2}, q^{-2 N_{1}-2}, q^{-2 f_{1}-2 f_{2}-2},-p^{-1} q^{2 f_{1}+2 f_{2}-2 N_{2}} ; q^{2}\right) .
$$

The proof of Proposition 5.3 is similar to the proof of Proposition 4.3. Proposition 5.3 can also be obtained from Proposition 4.3 using the substitutions as indicated earlier. It can also be obtained by using $e_{0}^{N}=\sum C_{n_{1}, n_{2}, 0}^{N_{1}, N} e_{n_{1}}^{N_{1}} \otimes e_{n_{2}}^{N_{2}}$, Propositions 5.2 and 5.1 and the explicit value for the Clebsch-Gordan coefficients for $n=0, N=N_{1}+N_{2}-2 j$,

$$
C_{n_{1}, n_{2}, 0}^{N_{1}, N_{2}, N}=(-1)^{n_{2}} q^{n_{2}\left(N_{2}-j-1\right)} \sqrt{\frac{\left(q^{2 n_{1}+2} ; q^{2}\right)_{n_{2}}\left(q^{2 N_{1}-2 n_{1}} ; q^{-2}\right)_{n_{2}}}{\left(q^{2} ; q^{2}\right)_{n_{2}}\left(q^{2 N_{2}} ; q^{-2}\right)_{n_{2}}}} \sqrt{\frac{\left(q^{2 N_{2}} ; q^{-2}\right)_{j}}{\left(q^{2 N_{1}+N_{2}-2 j+2} ; q^{2}\right)_{j}}} .
$$

See e.g. $[24, \S 14.3]$, but this simple case can also be derived as follows. Apply $C$ to both sides of (5.1) to obtain a three-term recurrence for the Clebsch-Gordan coefficients, which reduces to a two-term recurrence for $n=0$. This can be easily solved, with the initial condition following from the unitarity and the normalisation, see [23] for a similar derivation. Then we have a sum involving the product of two dual $q$-Krawtchouk polynomials. Upon inserting the series representation we obtain a triple sum, and after interchanging summations we can use the $q$-binomial theorem and the $q$-Chu-Vandermonde sum, see [10], to obtain a single ${ }_{4} \varphi_{3}$-series. 
Remark 5.4. With Proposition 5.3 at hand it is straightforward to calculate the $U_{q}(\mathfrak{s u}(2))$ counterparts of Theorems 4.5 and 4.10. The Al-Salam and Chihara, respectively AskeyWilson, polynomials have to be replaced by dual $q$-Krawtchouk, respectively $q$-Racah, polynomials. The result can also be obtained from Theorems 4.5 and 4.10 by substitution as indicated earlier, so we do not give them explicitly. These formulas give an alternative for the formulas of Groza and Kachurik [12].

In the representation space $W_{N_{1}} \otimes W_{N_{2}}$ we have two bases of eigenvectors for the action of $X_{p} A$, namely $\phi_{f}^{N}$ and $\phi_{f_{1}, f_{2}}^{N_{1}, N_{2}}$, and the corresponding Clebsch-Gordan coefficients are given by Proposition 5.3, since, with $N=N_{1}+N_{2}-2 j$,

$$
\begin{aligned}
& \phi_{f_{1}, f_{2}}^{N_{1}, N_{2}}=\sum_{j=0}^{\min \left(N_{1}, N_{2}\right)} \sum_{n=0}^{N}\left\langle\phi_{f_{1}, f_{2}}^{N_{1}, N_{2}}, e_{n}^{N}\right\rangle e_{n}^{N} \\
& =\sum_{j=0}^{\min \left(N_{1}, N_{2}\right)}\left\langle\phi_{f_{1}, f_{2}}^{N_{1}, N_{2}}, e_{0}^{N}\right\rangle \sum_{n=0}^{N} r_{n}\left(q^{2 j-2 f_{1}-2 f_{2}}-q^{2 f_{1}+2 f_{2}-2 j-2 N} / p ; p, N ; q^{2}\right) e_{n}^{N} \\
& =\sum_{j=0}^{\min \left(N_{1}, N_{2}\right)}\left\langle\phi_{f_{1}, f_{2}}^{N_{1}, N_{2}}, e_{0}^{N}\right\rangle \phi_{f_{1}+f_{2}-j}^{N} .
\end{aligned}
$$

Here we use the convention that $\phi_{f}^{N}=0$ for $f>N$ or $f<0$. So introducing the notation

$$
\phi_{f_{1}, f_{2}}^{N_{1}, N_{2}}=\sum_{f, j} C_{f_{1}, f_{2}, f}^{N_{1}, N_{2}, N}(p) \phi_{f}^{N},
$$

we see that the Clebsch-Gordan coefficients are zero unless $f_{1}+f_{2}=f+j$, and then $C_{f_{1}, f_{2}, f}^{N_{1}, N_{2}, N}(p)=\left\langle\phi_{f_{1}, f_{2}}^{N_{1}, N_{2}}, e_{0}^{N}\right\rangle$. So, by Proposition 5.3 we have proved that the $q$-Racah polynomials occur as Clebsch-Gordan coefficients for $U_{q}(\mathfrak{s u}(2))$.

Using (5.3) in a special case we can obtain the linearisation coefficients for the two parameter family of Askey-Wilson polynomials occurring as spherical functions on the quantum $S U(2)$ group, cf. [21]. We consider odd-dimensional representations; $N_{1}=2 l_{1}$, $N_{2}=2 l_{2}, l_{1}, l_{2} \in \mathbb{Z}_{+}$. Then the kernel of $t^{2 l_{1}}\left(X_{p} A\right)$ is one dimensional and spanned by $\phi_{l_{1}}^{2 l_{1}}(p)$. Moreover, $\phi_{l_{1}, l_{1}}^{2 l_{1}, 2 l_{2}}(p)=\phi_{l_{1}}^{2 l_{1}}(p) \otimes \phi_{l_{2}}^{2 l_{2}}(p)$. Next we consider matrix elements as linear functionals on $U_{q}(\mathfrak{s u}(2))$ to find

$$
\begin{aligned}
& \sum_{(X)}\left\langle t^{2 l_{1}}\left(X_{(1)}\right) \phi_{l_{1}}^{2 l_{1}}(p), \phi_{l_{1}}^{2 l_{1}}(r)\right\rangle\left\langle t^{2 l_{2}}\left(X_{(2)}\right) \phi_{l_{2}}^{2 l_{2}}(p), \phi_{l_{2}}^{2 l_{2}}(r)\right\rangle \\
& =\left\langle t^{2 l_{1}} \otimes t^{2 l_{2}}(\Delta(X)) \phi_{l_{1}, l_{1}}^{2 l_{1}, 2 l_{2}}(p), \phi_{l_{1}, l_{1}}^{2 l_{1}, 2 l_{2}}(r)\right\rangle \\
& =\sum_{l=\left|l_{1}-l_{2}\right|}^{l_{1}+l_{2}} C_{l_{1}, l_{2}, l}^{2 l_{1}, 2 l_{2}, 2 l}(p) C_{l_{1}, l_{2}, l}^{2 l_{1}, 2 l_{2}, 2 l}(r)\left\langle t^{2 l}(X) \phi_{l}^{2 l}(p), \phi_{l}^{2 l}(r)\right\rangle,
\end{aligned}
$$

where $r>0$ is another parameter and $\Delta(X)=\sum_{(X)} X_{(1)} \otimes X_{(2)}$. 
The dual Hopf *-algebra $A_{q}(S U(2))$ generated by the matrix elements of the representations $t^{N}, N \in \mathbb{Z}_{+}$, of $U_{q}(\mathfrak{s u}(2))$, is known in terms of generators and relations, cf. [6], [17], [21]. Koornwinder [21] has given an explicit expression for the element in $A_{q}(S U(2))$ corresponding to the linear functionals considered in (5.4);

$$
\left\langle t^{2 l}(X) \phi_{l}^{2 l}(p), \phi_{l}^{2 l}(r)\right\rangle=\frac{q^{-l}}{\left(q^{2 l+2} ; q^{2}\right)_{l}}\left\langle X, p_{l}\left(\rho ; q \sqrt{\frac{p}{r}}, q \sqrt{\frac{r}{p}}, \frac{-q}{\sqrt{p r}},-q \sqrt{p r} \mid q^{2}\right)\right\rangle,
$$

where $\rho \in A_{q}(S U(2))$ is some fixed simple element, which is, up to an affine scaling, the linear functional $X \mapsto\left\langle t^{2}(X) \phi_{1}^{2}(p), \phi_{1}^{2}(r)\right\rangle$, and the last $\langle\cdot, \cdot\rangle$ denotes the duality between $U_{q}(\mathfrak{s u}(2))$ and $A_{q}(S U(2))$. Since $A_{q}(S U(2))$ is the dual Hopf $*$-algebra, the left hand side of (5.4) corresponds to the multiplication of the two linear functionals. So (5.4) leads to the following identity in $A_{q}(S U(2))$;

$$
p_{l_{1}}(\rho) p_{l_{2}}(\rho)=\sum_{l=\left|l_{1}-l_{2}\right|}^{l_{1}+l_{2}} q^{l_{1}+l_{2}-l} \frac{\left(q^{2 l_{1}+2} ; q^{2}\right)_{l_{1}}\left(q^{2 l_{2}+2} ; q^{2}\right)_{l_{2}}}{\left(q^{2 l+2} ; q^{2}\right)_{l}} C_{l_{1}, l_{2}, l}^{2 l_{1}, 2 l_{2}, 2 l}(p) C_{l_{1}, l_{2}, l}^{2 l_{1}, 2 l_{2}, 2 l}(r) p_{l}(\rho)
$$

with $p_{l}(\cdot)=p_{l}\left(\cdot ; q \sqrt{\frac{p}{r}}, q \sqrt{\frac{r}{p}}, \frac{-q}{\sqrt{p r}},-q \sqrt{p r} \mid q^{2}\right)$.

The only information on $A_{q}(S U(2))$ needed is the existence of a family of onedimensional representations sending $\rho$ to $\cos \theta$. Thus, applying the one-dimensional representations of $A_{q}(S U(2))$ and using Proposition 5.3 proves the following linearisation coefficient formula.

Theorem 5.5. Let $p_{l}(x)=p_{l}\left(x ; q \sqrt{\frac{p}{r}}, q \sqrt{\frac{r}{p}}, \frac{-q}{\sqrt{p r}},-q \sqrt{p r} \mid q^{2}\right), p, r>0$, be defined in terms of Askey-Wilson polynomials (4.6). Then the coefficients in the linearisation formula

$$
p_{l_{1}}(x) p_{l_{2}}(x)=\sum_{j=0}^{2 \min \left(l_{1}, l_{2}\right)} c_{j} p_{l_{1}+l_{2}-j}(x)
$$

are given by a product of two balanced terminating ${ }_{4} \varphi_{3}$-series;

$$
\begin{aligned}
c_{j}= & q^{-j(j-1)} q^{j+4 j l_{1}}\left(q^{2 l_{1}+2} ; q^{2}\right)_{l_{1}-j}\left(q^{2 l_{2}+2} ; q^{2}\right)_{l_{2}}\left[\begin{array}{c}
2 l_{2} \\
j
\end{array}\right]_{q^{2}} \\
& \times \frac{\left(q^{2 l_{1}+2 l_{2}} ; q^{-2}\right)_{j}}{\left(q^{2 l_{1}+2 l_{2}+2} ; q^{2}\right)_{1}+l_{2}-j} \frac{1-q^{4 l_{1}+4 l_{2}-4 j+2}}{1-q^{4 l_{1}+4 l_{2}-2 j+2}} \\
& \times p^{j / 2}\left(-p^{-1} q^{-2 l_{1}-2 l_{2}} ; q^{2}\right)_{j}{ }_{4} \varphi_{3}\left(\begin{array}{c}
q^{-2 j}, q^{-2 l_{2}}, q^{2 j-2-4 l_{1}-4 l_{2}},-p^{-1} q^{-2 l_{2}} \\
q^{-4 l_{2}}, q^{-2 l_{1}-2 l_{2}},-p^{-1} q^{-2 l_{1}-2 l_{2}} ; q^{2}, q^{2}
\end{array}\right) \\
& \times r^{j / 2}\left(-r^{-1} q^{-2 l_{1}-2 l_{2}} ; q^{2}\right)_{j}{ }_{4} \varphi_{3}\left(\begin{array}{c}
q^{-2 j}, q^{-2 l_{2}}, q^{2 j-2-4 l_{1}-4 l_{2}},-r^{-1} q^{-2 l_{2}} \\
q^{-4 l_{2}}, q^{-2 l_{1}-2 l_{2}},-r^{-1} q^{-2 l_{1}-2 l_{2}} ; q^{2}
\end{array}\right) .
\end{aligned}
$$

Remark 5.6. (i) In particular, for $p=r$ the linearisation coefficients are positive. This can already be observed without the explicit knowledge of the linearisation coefficients, see [17, $\S 8.3],[19, \S 7]$. 
(ii) For $p=r=1$ the Askey-Wilson polynomials $p_{l}\left(x ; q, q,-q,-q \mid q^{2}\right)$ are the continuous $q$-Legendre polynomials $C_{l}\left(x ; q^{2} \mid q^{4}\right)$, see $[3, \S 4]$. This is a special case of the continuous $q$-ultraspherical polynomials introduced by Rogers at the end of the 18th century. Rogers calculated the linearisation coefficients for the continuous $q$-ultraspherical polynomials, see e.g. $[3, \S 4],[10, \S 8.5]$, and we can go from Theorem 5.5 to the special case of Rogers's result by using Andrew's summation formula, see [10, (II.17)].

\section{REFERENCES}

1. W.A. Al-Salam, Characterization theorems for orthogonal polynomials, 'Orthogonal Polynomials: Theory and Practice' (P. Nevai, ed.), NATO ASI series C, vol. 294, Kluwer, 1990, pp. 1-24.

2. W.A. Al-Salam and T.S. Chihara, Convolutions of orthonormal polynomials, SIAM J. Math. Anal. 7 (1976), 16-28.

3. R. Askey and J. Wilson, Some basic hypergeometric orthogonal polynomials that generalize Jacobi polynomials, Mem. Amer. Math. Soc. 54 (1985), no. 319.

4. J.M. Berezanskiu, Expansions in Eigenfunctions of Selfadjoint Operators, Transl. Math. Monographs 17, Amer. Math. Soc., 1968.

5. I.M. Burban and A.U. Klimyk, Representations of the quantum algebra $U_{q}\left(s u_{1,1}\right)$, J. Phys. A: Math. Gen. 26 (1993), 2139-2151.

6. V. Chari and A. Pressley, A Guide to Quantum Groups, Cambridge Univ. Press, 1994.

7. C.F. Dunkl, A difference equation and Hahn polynomials in two variables, Pacific J. Math. 92 (1981), $57-71$.

8. __ Orthogonal polynomials with symmetry of order three, Canad. J. Math. 36 (1984), 685-717.

9. A. Erdélyi, W. Magnus, F. Oberhettinger, F.G. Tricomi, Higher Transcendental Functions, Vol. 2, McGraw-Hill, 1953.

10. G. Gasper and M. Rahman, Basic Hypergeometric Series, Cambridge Univ. Press, 1990.

11. Y.I. Granovskii and A.S. Zhedanov, New construction of $3 n j$-symbols, J. Phys A: Math. Gen. 26 (1993), 4339-4344.

12. V.A. Groza and I.I. Kachurik, Addition and product theorems for Krawtchouk, Hahn and Racah qpolyomials, Doklady Akad. Nauk Ukraine SSR, Ser. A 89, 3-6, (in Russian).

13. E.G. Kalnins, H.L. Manocha and W. Miller, Models of q-algebra representations: tensor products of special unitary and oscillator algebras, J. Math. Phys. 33 (1992), 2365-2383.

14. A.U. Klimyk and I.I. Kachurik, Spectra, eigenvectors and overlap functions for representation operators of q-deformed algebras, Commun. Math. Phys. 175 (1996), 89-111.

15. R. Koekoek and R.F. Swarttouw, The Askey-scheme of hypergeometric orthogonal polynomials and its $q$-analogue, Report 94-05, Technical University Delft (1994), available from ftp.twi.tudelft.nl in directory /pub/publications/tech-reports.

16. H.T. Koelink, On Jacobi and continuous Hahn polynomials, Proc. Amer. Math. Soc. 124 (1996), $887-898$.

17. Askey-Wilson polynomials and the quantum SU(2) group: survey and applications, Acta Appl. Math. (to appear).

18. T.H. Koornwinder, Meixner-Pollaczek polynomials and the Heisenberg algebra, J. Math. Phys. 30 (1989), 767-769.

19. - Positive convolution structures associated with quantum groups, 'Probability Measures on Groups X' (H. Heyer, ed.), Plenum, 1991, pp. 249-268.

20. _ Askey-Wilson polynomials for root systems of type BC, Contemp. Math. 138 (1992), $189-204$.

21. _ Askey-Wilson polynomials as zonal spherical functions on the SU(2) quantum group, SIAM J. Math. Anal. 24 (1993), 795-813.

22. D.R. Masson and J. Repka, Spectral theory of Jacobi matrices in $\ell^{2}(\mathbb{Z})$ and the su $(1,1)$ Lie algebra, SIAM J. Math. Anal. 22 (1991), 1133-1146.

23. J. Van der Jeugt, Coupling coefficients for Lie algebra representations and addition formulas for special functions, preprint (1996). 
24. N.J. Vilenkin and A.U. Klimyk, Representation of Lie Groups and Special Functions, 3 volumes, Kluwer, 1991, 1993.

Vakgroep Wiskunde, Universiteit van Amsterdam, Plantage Muidergracht 24, 1018 TV Amsterdam, the Netherlands

E-mail address: koelink@fwi.uva.nl

Vakgroep Toegepaste Wiskunde en Informatica, Universiteit Gent, Krijgslaan 281-S9, B-9000 Gent, Belgium

E-mail address: Joris.VanderJeugt@rug.ac.be 